\title{
Revealing Network Structure, Confidentially: Improved Rates for Node-Private Graphon Estimation
}

\author{
Christian Borgs* $\quad$ Jennifer Chayes* $\quad$ Adam Smith $^{\dagger} \quad$ Ilias Zadik ${ }^{\ddagger}$
}

\begin{abstract}
Motivated by growing concerns over ensuring privacy on social networks, we develop new algorithms and impossibility results for fitting complex statistical models to network data subject to rigorous privacy guarantees. We consider the so-called node-differentially private algorithms, which compute information about a graph or network while provably revealing almost no information about the presence or absence of a particular node in the graph.

We provide new algorithms for node-differentially private estimation for a popular and expressive family of network models: stochastic block models and their generalization, graphons. Our algorithms improve on prior work [15], reducing their error quadratically and matching, in many regimes, the optimal nonprivate algorithm [37]. We also show that for the simplest random graph models $(G(n, p)$ and $G(n, m))$, node-private algorithms can be qualitatively more accurate than for more complex models - converging at a rate of $\frac{1}{\varepsilon^{2} n^{3}}$ instead of $\frac{1}{\varepsilon^{2} n^{2}}$. This result uses a new extension lemma for differentially private algorithms that we hope will be broadly useful.
\end{abstract}

\section{Introduction}

Network data play an increasingly important role in many scientific fields. Data from social networks, in which the nodes represent individuals and edges represent relationships among them, are transforming sociology, marketing, and political science, among others. However, what makes these data so valuable also makes them highly sensitive - consider, for example, the public sentiment surrounding the recent Cambridge Analytica scandal.

What kinds of information can we release about social networks while preserving the privacy of their users? Straightforward approaches, such as removing obvious identifiers or releasing summaries that concern at least a certain number of nodes, can be easily broken [46, 38].

In this paper, we develop new algorithms and impossibility results for fitting complex statistical models to network data subject to rigorous privacy guarantees. We consider differentially private algorithms [23]. There are two main variants of differential privacy for graphs: edge and node differential privacy [50]. Intuitively, edge differential privacy ensures that an algorithm's output does not reveal the inclusion or removal of a particular edge in the graph, while node differential privacy hides the inclusion or removal of a node together with all its adjacent edges. Edge privacy is weaker (hence easier to achieve) and has been studied more extensively [47, 50, 34, 45, 43, 35, 28, 29, 33, 40, 32, 27, 7, 45, 35, 43, 32, 55].

${ }^{*}$ Microsoft Research New England. \{Christian.Borgs, Jennifer. Chayes\}@microsoft.com.

${ }^{\dagger}$ Boston University. ads22@bu.edu.

${ }^{\ddagger}$ MIT. izadik@mit.edu. Research done in part while an intern at Microsoft Research New England. 
We study node-differentially private algorithms. These ensure that, no matter what an analyst observing the output knows ahead of time, she learns the same things about an individual Alice regardless of whether Alice's data are used or not. Node privacy's stringency makes the design of accurate, private algorithms challenging; only a small number of techniques for designing such algorithms are known [36, 8, 18, 49, 21].

We provide new algorithms for node-differentially private estimation for a popular and expressive family of network models: stochastic block models and their generalization, graphons. Our algorithms improve on prior work (by a subset of us [15]), roughly reducing their error quadratically and matching, in many regimes, the optimal nonprivate algorithm [37, 44]. We also show that for the simplest random graph models $(G(n, p)$ and $G(n, m))$, node-private algorithms can be qualitatively more accurate than for more complex models - converging at a rate of $\frac{1}{\varepsilon^{2} n^{3}}$ instead of $\frac{1}{\varepsilon^{2} n^{2}}$. This result uses a new extension lemma for differentially private algorithms that we hope will be broadly useful.

Modeling Large Graphs via Graphons Traditionally, large graphs have been modeled using various parametric models, one of the most popular being the stochastic block model [30]. Here one postulates that an observed graph was generated by first assigning vertices at random to one of $k$ groups, and then connecting two vertices with a probability that depends on the groups the two vertices are members of.

As the number of vertices of the graph in question grows, we do not expect the graph to be well described by a stochastic block model with a fixed number of blocks. We therefore consider nonparametric models described by a graphon. A graphon is a measurable, bounded function $W:[0,1]^{2} \rightarrow[0, \infty)$ such that $W(x, y)=W(y, x)$, which for convenience we take to be normalized: $\int W=1$. Given a graphon, we generate a graph on $n$ vertices by first assigning i.i.d. uniform labels $x_{i} \in[0,1], i=1,2, \ldots, n$ to the vertices, and then connecting vertices $i, j$ with labels $x_{i}, x_{j}$ with probability $H_{n}(i, j)=\rho_{n} W\left(x_{i}, x_{j}\right)$, where $\rho_{n}$ is a parameter determining the density of the generated graph $G_{n}$ with $\rho_{n}\|W\|_{\infty} \leq 1$. We call $G_{n}=G_{n}(\rho W)$ a $W$-random graph with target density $\rho_{n}$ (or simply a $\rho_{n} W$-random graph).

This model captures stochastic block models as well as more complex models, e.g. random geometric graphs, where each vertex corresponds to a point in a metric space (selected randomly according to a particular distribution) and vertices share an edge if their points are sufficiently close [26, 20, 48, 24].

For both the "dense" setting (where the target density $\rho_{n}$ does not depend on the number of vertices) and the "sparse" setting (where $\rho_{n} \rightarrow 0$ as $n \rightarrow \infty$ ), graphons play a key role in the convergence theory for graph sequences [31, 4, 42, 9, 10, 12, 13, 14], providing limit objects in several natural topologies.

Metrics for Estimation Given a single graph $G_{n}$ generated as $\rho W$-random for unknown $\rho$ and $W$, how well can we estimate $\rho$ and $W$ ? This task has now been studied extensively $5,51,19,6$, 41, 53, 39, 54, 16, 3, 56, 25, 2, 17, 1, 37, 44]. One issue faced by all these works is identifiability: multiple graphons can lead to the same distribution on $G_{n}$. Specifically, two graphons $W$ and $\tilde{W}$ lead to the same distribution on $W$-random graphs if and only if there are measure preserving $\operatorname{maps} \varphi, \tilde{\varphi}:[0,1] \rightarrow[0,1]$ such that $W^{\varphi}=\widetilde{W^{\widetilde{\varphi}}}$, where $W^{\varphi}$ is defined by $W(x, y)=W(\varphi(x), \varphi(y))$ [22, 11]. Hence, there is no "canonical graphon" that an estimation procedure can output. Some of the literature circumvents identifiability by making strong additional assumptions that imply the 
existence of canonical equivalence class representatives. We make no such assumptions, but instead define consistency in terms of a metric on equivalence classes. We use a variant of the $L_{2}$ metric,

$$
\delta_{2}\left(W, W^{\prime}\right)=\inf _{\varphi:[0,1] \rightarrow[0,1]}\left\|W^{\varphi}-W^{\prime}\right\|_{2},
$$

where $\varphi$ ranges over measure-preserving bijections.

In this work, we set aside questions of computational efficiency and focus on establishing what rates are possible in principle (our algorithms, like the nonprivate state of the art, run in time roughly exponential in $n$ ).

For our purposes, the most relevant work is that of Klopp, Tzybakov and Verzalen [37], who establish tight upper and (in parallel to [44]) lower bounds on the error rate of nonprivate algorithms, given a single $n$-vertex $\rho W$-random graph and a target number of blocks, $k$. Our algorithms match their rate for large enough values of the privacy parameter.

Private Algorithms for Graph Data and the Rewiring Metric Let $\mathcal{A}$ be a randomized algorithm that takes values from some input metric space $(\mathcal{M}, d)$ (called the space of data sets) and ouputs probability distributions on some measurable space $(\Omega, \mathcal{F})$.

Definition 1.1. The algorithm $\mathcal{A}$ is $\varepsilon$-differential private $(\varepsilon-D P)$ with respect to the metric $d$ if, for all subsets $S \in \mathcal{F}$ and $D_{1}, D_{2} \in \mathcal{M}$,

$$
\mathbb{P}\left(\mathcal{A}\left(D_{1}\right) \in S\right) \leq \exp \left[\varepsilon d\left(D_{1}, D_{2}\right)\right] \mathbb{P}\left(\mathcal{A}\left(D_{2}\right) \in S\right) .
$$

The metric $d$ is typically defined by specifying pairs of data sets that are adjacent (i.e., at distance 1 from each other), and then letting $d$ be the induced path metric.

There are two natural variants of differential privacy suited for graph datasets, edge differential privacy and node differential privacy. Intuitively, edge differentially private algorithms hide the presence or absence of a particular relationship between individuals in a social network, while node differentially private algorithms protect each individual together with all his/her relationsips. In both cases, the data set is an undirected graph with no self-loops; we let $\mathcal{G}_{n}$ denote the set of such graphs on $n$ vertices. Formally, edge differential privacy is obtained by taking $d$ to count the number of edges that differ between two graphs (the Hamming metric on adjacency matrices). In contrast, node differential privacy is defined with respect to the rewiring metric, or node distance, between graphs: we say that two distinct graphs $G, G^{\prime}$ are at node-distance 1 (or adjacent) if one can be obtained from the other by inserting or removing arbitrary sets of edges adjacent to a singe vertex, a process we call rewiring the vertex. For arbitrary $G_{1}, G_{2} \in \mathcal{G}_{n}$, define the node-distance between them, $d_{v}\left(G_{1}, G_{2}\right)$, to be the minimum number of vertices of $G_{1}$ that need to be rewired to obtain $G_{2}$. A randomized algorithm $\mathcal{A}$ defined on $\mathcal{G}_{n}$ is $\varepsilon$-node differentially private ( $\varepsilon$-node DP) if it is $\varepsilon$-differentially private with respect to the node-distance $d_{v}$.

Edge differential privacy is a weaker notion and has been extensively studied over the past decade. Algorithms have been developed for various tasks such as the release of subgraph counts, the degree distribution and the parameters of generative graph models [28], 35], [45], 33], 34], [47]. On the other hand, the node-differential privacy is a much stronger privacy guarantee. The first nontrivial node-differentially algorithms were designed (concurrently) in [8, 18, 36], with a focus on algorithms that release one-dimensional summaries of a network such as subgraph counts. Later work [49, 15, 21] introduced higher-dimensional techniques. Most relevant here, a subset of 
us gave the first algorithms for node-private graphon estimation [15]. A common thread to all these works is the use of Lipschitz extensions in the rewiring metric to control the sensitivity of summary statistics for sparse graphs. A key piece of this paper is a novel use of such extensions.

The previous results for graphon estimation achieved estimation error going to 0 for a large parameter range, but fell short in several respects: first, even when $\varepsilon$ is arbitrarily large, the algorithm does not match the best nonprivate bounds. Secondly, there was no evidence that the extra terms due to privacy (involving $\varepsilon$ ) in the accuracy guarantee were necessary.

\subsection{Contributions}

New Upper Bounds for Estimating $k$-Block Graphons Our main focus is the problem of estimating a bounded normalized graphon $W$ via a node-differentially private algorithm. The estimation algorithm observes one sample of a $\rho W$-random graph, and outputs the description of a graphon $\hat{W}$ that it hopes is close to $W$. We consider algorithms that output a graphon with a succinct description-namely, we assume the estimate $\hat{W}$ is a $k$-block graphon with equal-weight blocks (such a graphon can be described by a $k \times k$ symmetric matrix). The parameter $k$ offers a regularization of sorts, trading off the model's expressivity for complexity. We measure the algorithm's error by the expected squared $\delta_{2}$ distance (see (1.1) ) between $\hat{W}$ and $W$. Borgs et al. [15] studied this problem, developing an inefficient estimation procedure (henceforth the "BCS" algorithm [15, Algorithm 1]) and establishing an upper bound on its error.

Our first contribution is a new analysis of the BCS algorithm that significantly improves the error bound, matching the (tight) nonprivate bounds for a large range of parameters. The new and old results can be summarized as the following upper bound on the mean squared error $\mathbb{E}\left[\delta_{2}(\hat{W}, W)^{2}\right]$ of the following form.

Theorem 1 (Informal). Fix some $k \geq 1$ and let $\mathcal{A}$ be the BCS algorithm. Then for all bounded graphons $W$,

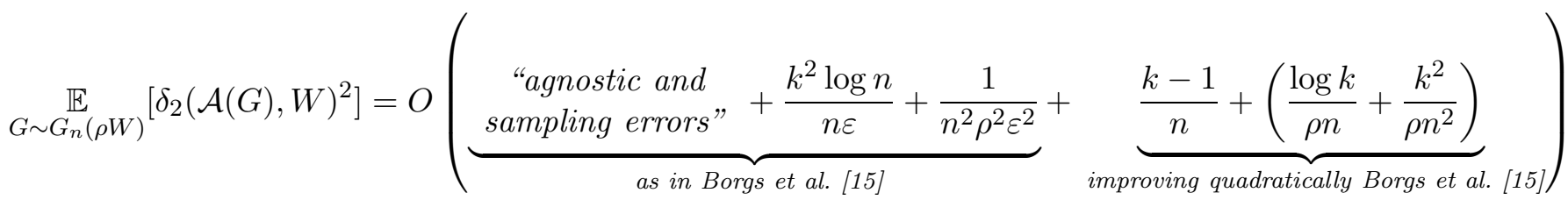

Here, the phrase "agnostic and sampling errors" covers two terms that are present in both bounds. The "agnostic error" corresponds to the distance from the true graphon $W$ to the nearest $k$-block graphon - a model misspecification error. It is unavoidable for algorithms that output $k$-block graphons. The "sampling" term corresponds to the expected distance between the true graphon $W$ and the probability matrix $\left(W\left(x_{i}, x_{j}\right)\right)_{i, j=1}^{n}$ defining the $\rho W$-random graph. This distance is a random variable that can be bounded in different ways depending on what is known about $W$. If $W$ is itself a $k$-block graphon, then the the agnostic error is 0 , and the sampling error (about $\sqrt{k / n}$ with high probability) is subsumed by the other error terms.

Notice that our improvement to the accuracy bound lies in the "non-private" (that is, independent of $\varepsilon$ ) part of the error.

This nonprivate part of our new bound is in fact optimal, as it matches the lower bounds for nonprivate algorithms. Specifically, consider the case that the true graphon $W$ is in fact a $k$-block 
graphon and define the rate

$$
R_{k}(\rho, \varepsilon, n)=\min _{\substack{\mathcal{A} \\ \varepsilon-\text { node-DP }}} \max _{W k-\text { block }} \underset{G \sim G_{n}(\rho W)}{\mathbb{E}}\left[\delta_{2}(\mathcal{A}(G), W)^{2}\right] .
$$

Klopp et al. 37, Prop. 3.4] (and McMillan and Smith [44, Theorem 3]) establish the best rate if we allow any estimation algorithm $\mathcal{A}$-private or not- to be

$$
\Theta\left(\min \left\{\sqrt{\frac{k}{n}}+\left(\frac{\log k}{\rho n}+\frac{k^{2}}{\rho n^{2}}\right), 1\right\}\right) \text { for } k \geq 2 .
$$

In particular, focusing on $\varepsilon$-node-DP algorithms we conclude that for any $k \geq 2$,

$$
R_{k}(\rho, \varepsilon, n)=\Omega\left(\min \left\{\sqrt{\frac{k}{n}}+\left(\frac{\log k}{\rho n}+\frac{k^{2}}{\rho n^{2}}\right), 1\right\}\right)
$$

Notice that our upper bound as established in Theorem 1 matches exactly this lower bound when the true graphon has exactly $k$ blocks and $\varepsilon$ is sufficiently large (since then the agnostic error is 0 , the sampling error is know to be $O(\sqrt{k / n})$, and the $\varepsilon$-dependent terms go to 0$)$. In particular, using Theorem 1 we conclude a tight characterization of the $\varepsilon$-independent part of the rate $R_{k}(\rho, \varepsilon, n)$,

Collorary 1 (Informal). Fix some $k \geq 2$. Then there exists an algorithm such that for all bounded graphons $W$,

$$
R_{k}(\rho, \varepsilon, n)=O\left(\frac{k^{2} \log n}{n \varepsilon}+\frac{1}{n^{2} \rho^{2} \varepsilon^{2}}\right)+\underbrace{O\left(\sqrt{\frac{k-1}{n}}+\left(\frac{\log k}{\rho n}+\frac{k^{2}}{\rho n^{2}}\right)\right)}_{\text {tight nonprivate part based on 1.2) }}
$$

Additional Error Due to Privacy $(k \geq 2)$ To understand whether we have found the true minimax rate, it remains to understand whether the terms based on $\varepsilon$ are optimal. We show that the second of these cannot be improved, on the slightly less restrictive case where the blocks of the $k$-block graphon can have different sizes, a set we denote by $\tilde{W}[k]$.

Theorem 2 (Informal). For $k \geq 2$,

$$
\tilde{R}_{k}(\rho, \varepsilon, n)=\Omega\left(\frac{1}{n^{2} \varepsilon^{2}}\right)
$$

where $\tilde{R}_{k}(\rho, \varepsilon, n)$ is defined to be

$$
\min _{\mathcal{A} \varepsilon-\text { node-DP }} \max _{W \in \tilde{W}[k]} \mathbb{E}_{G \sim G_{n}(\rho W)}\left[\delta_{2}(\mathcal{A}(G), W)^{2}\right] .
$$

This lower bound applies even to algorithms that simply estimate the unknown density parameter $\rho$. The proof of this lower bound is fairly simple, relying on the fact that even if the connection probabilities of a 2-block graphon are known, estimating the graphon requires one to accurately estimate the probability mass of the two blocks. We reduce to this latter problem from the well-studied problem of estimating the bias of a sequence of $n$ coin flips differentially privately.

We leave open the question of whether the term $\frac{k^{2} \log n}{n \varepsilon}$ is necessary. 
The Case of Erdös-Renyi Graphs (1-Block Graphons) The upper bounds above all apply for $k=1$, in particular, but the lower bounds generally do not yield anything interesting in that case. The case of $k=1$ corresponds to graphs generated according to the well-studied Erdös-Renyi model, where each possible edge appears independently with an unknown probability $p$. To phrase this as an estimation problem, consider the scale parameter $\rho$ to be known, and the algorithm's goal is to estimate a constant graphon $W(x, y)=p$ subject to $p \leq \rho$. (Unlike in the case of larger $k$, estimating the normalized graphon $W$ is trivial since, after normalization, $W(x, y)=1$.)

Nonprivately, the optimal estimator is the edge density of the observed graph, (\#edges) $/\left(\begin{array}{l}n \\ 2\end{array}\right)$.

What about private algorithms? First, observe that the algorithm $\mathcal{A}_{0}$ that adds Laplace noise of order $\frac{1}{n \varepsilon}$ to the edge density is $\varepsilon$-node differentially private. Furthermore, for $p \in[0, \rho]$,

$$
\mathbb{E}_{G \sim G_{n, p}}\left[\left|\mathcal{A}_{0}(G)-p\right|^{2}\right]=O\left(\frac{\rho}{n^{2}}+\frac{1}{n^{2} \varepsilon^{2}}\right) .
$$

Potentially surprisingly, we establish that the rate obtained this way is not optimal. As we explain in section 3, the main reason for the suboptimality of this method is that it is based on calculating the worst-case sensitivity of the edge density over the space of all undirected graphs. In particular, this estimator ignores the rich structure of the Erdos-Renyi graphs. Using this structure, we establish a series of results relating the node-distance and the Erdos-Renyi graphs (Lemma 9.3, Lemma 9.4) along-side with a general extension result (Proposition 5.1) which combined allows to prove the following improved upper bound

Theorem 3 (Informal). There exists an $\varepsilon$-node-DP algorithm $\mathcal{A}$ such that for any $\rho \in(0,1]$,

$$
\max _{p \in[0, \rho]} \mathbb{E}_{G \sim G_{n, p}}\left[(A(G)-p)^{2}\right]=O\left(\frac{\rho}{n^{2}}+\frac{\log n}{n^{3} \varepsilon^{2}}\right) .
$$

Using the same techniques we are able to establish the corresponding result for the uniform $G(n, m)$ model which obtains an error

$$
O\left(\frac{\log n}{n^{3} \varepsilon^{2}}\right)
$$

avoiding the edge-density variance term which appears in the Erdos-Renyi case, $\frac{\rho}{n^{2}}$. We end this section with a novel lower bound for $G(n, m)$ model.

Theorem 4 (Informal). Suppose $\varepsilon$ is a constant. Then,

$$
\min _{\mathcal{A} \varepsilon-\text { node-DP }} \max _{m \in\left[\frac{1}{3}\left(\begin{array}{c}
n \\
2
\end{array}\right), \frac{2}{3}\left(\begin{array}{c}
n \\
2
\end{array}\right)\right]} \mathbb{E}_{G \sim G(n, m)}\left[\left(A(G)-\frac{m}{\left(\begin{array}{c}
n \\
2
\end{array}\right)}\right)^{2}\right]=\Omega\left(\frac{1}{n^{3} \varepsilon^{2}}\right) .
$$

This Theorem establishes that the upper bound for the $G(n, m)$ model is optimal up-tologarithmic terms in the $\varepsilon$-constant regime and suggests the same for the Erdos-Renyi case.

A General Extension Result In Section 4, we present in detail the general extension result we used in Section 3 as it could be of independent interest. The extension result works for an arbitrary $\varepsilon$-differentially private algorithm which receives input from a metric space $(M, d)$ and outputs distributions of an arbitrary output measurable space $(\Omega, \mathcal{F})$. We establish that if there exists such an $\varepsilon$-differentially private algorithm $\hat{\mathcal{A}}$ defined only on a subset of the input space $\mathcal{H}$, the algorithm can be extended to an $2 \varepsilon$-differentially private algorithm $\mathcal{A}$ defined on the whole input space $M$ such that if the input $G \in \mathcal{H}$, the distributions of the output of $\hat{\mathcal{A}}(G)$ coincides with the distribution of $\mathcal{A}(G)$. 


\section{Notation and Preliminaries}

$k$-block Graphons For every $k \in \mathbb{N}$, we embed the set of $k \times k$ symmetric matrices into the space of graphons as following: let $\mathcal{P}_{k}=\left(I_{1}, \ldots, I_{k}\right)$ be the partition of $[0,1]$ into adjacent intervals of lengths $\frac{1}{k}$. For $A \in \mathbb{R}_{\geq 0}^{k \times k}$ define $W[A]$ to be the step function which equals $A_{i j}$ on $I_{i} \times I_{j}$, for every $i, j \in[k]$. We say a graphon $W$ is a $k$-block graphon if $W=W[A]$ for some $A \in \mathbb{R}_{\geq 0}^{k \times k}$ and denote by $\mathcal{W}[k]$ the space of $k$-block graphon.

Distances between Graphons For $A, B$ symmetric $n \times n$ matrices and a graphon $W$ we set for convenience $\delta_{2}(A, W)=\delta_{2}(W[A], W)$ and $\delta_{2}(A, B)=\delta_{2}(W[A], W[B])$, where $\delta_{2}$ is defined for two graphons in 1.1. Furthermore we focus also on the, in general larger than $\delta_{2}$, distance

$$
\hat{\delta}_{2}(A, W)=\inf _{\pi \in \mathcal{S}_{n}}\left\|W\left[A^{\pi}\right]-W\right\|_{2}
$$

where $\pi$ ranges over all permurations of $\{1,2, \ldots, n\}$ and for all $i, j \in[n], A_{i j}^{\pi}=A_{\pi(i), \pi(j)}$. $\delta_{2}$ is in principle smaller than $\hat{\delta}_{2}$ as it minimizes the $\ell_{2}$ distance over all measure-preserving transformations, while the latter distance minimizes only on such transformation that can be expressed as permutations of the rows and columns of the underlying matrix $A$.

We consider two fundamental types of errors of approximation of $W$.

The agnostic error, or oracle error, of approximating $W$ by a $k$-block graphon with respect to $\delta_{2}$ and $\hat{\delta}_{2}$,

$$
\varepsilon_{k}^{(O)}(W)=\min _{B} \delta_{2}(B, W)
$$

and

$$
\hat{\varepsilon}_{k}^{(O)}(W)=\min _{B} \hat{\delta}_{2}(B, W)
$$

where $B$ ranges over all matrices in $\mathbb{R}^{k \times k}$. The agnostic errors corresponds to the model mispecification errors of the statistical problem of estimating $W$ using a $k$-block graphon. We consider them as benchmarks for our approach, and the errors an "oracle" could obtain (hence the superscript $O)$.

Scale of agnostic error: For any bounded $W$, both $\varepsilon_{k}^{(O)}(W)$ and $\hat{\varepsilon}_{k}^{(O)}(W)$ tend to zero as $k \rightarrow$ $+\infty$ (see [15, Sec. 2] for details). Furthermore, if $W$ is $\alpha$-Holder continuous for some $\alpha \in(0,1)$, i.e. if for some $C>0,\left|W(x, y)-W\left(x^{\prime}, y^{\prime}\right)\right| \leq C \delta^{\alpha}$ if $\left|x-x^{\prime}\right|+\left|y-y^{\prime}\right| \leq \delta$, then both $\varepsilon_{k}^{(O)}(W)$ and $\hat{\varepsilon}_{k}^{(O)}(W)$ are of order $O\left(k^{-\alpha}\right)$.

The sampling error of approximating $W$ from $G=G_{n}(\rho W)$ with respect to $\hat{\delta_{2}}$,

$$
\varepsilon_{n}(W)=\hat{\delta}_{2}\left(H_{n}(W), W\right)
$$

Recall that the only information for $W$ in the observed graph $G$ comes from the edge probabilities $H_{n}(i, j)=\rho W\left(x_{i}, x_{j}\right)$ where $x_{i}$ are the iid uniform in [0,1] labels of the vertices. Intuitively, a large discrepancy between the edge probability matrix $H_{n}(W)$ and $W$ results in bad estimation of $W$ given $G$. Unlike the agnostic error, the sampling error is a random variable (depending on the assignment of nodes to "types" in $[0,1]$.)

Scale of sampling error: For any bounded $W, \varepsilon_{n}(W) \stackrel{P}{\longrightarrow} 0$ as $n \rightarrow+\infty$ [15, Lemma 1]. Furthermore, if additionally $W$ is a $k$-block graphon it can be established that $\varepsilon_{n}(W)=O\left(\sqrt[4]{\frac{k}{n}}\right)$ with probability tending to one as $n \rightarrow+\infty[15$, Appendix D]. Finally, if $W$ is $\alpha$-Holder continuous then $\varepsilon_{n}(W)=O\left(n^{-\frac{\alpha}{2}}\right)$ with probability tending to one as $n \rightarrow+\infty$. 


\section{Private Graphon Estimation}

Model Let $k, n \in \mathbb{N}$ with $k \leq n, \Lambda \geq 1$ and $\varepsilon>0$. Suppose $W$ is an unknown normalised graphon with $\|W\|_{\infty} \leq \Lambda$. For some unknown "sparsity level" $\rho=\rho_{n} \in(0,1)$ with $\rho \Lambda \leq 1$, the analyst observes a graph $G$ sampled from the $\rho W$-random graph, $G_{n}(\rho W)$. The analyst's goal is to use an $\varepsilon$-node-DP algorithm $\mathcal{A}$ on $G$ to output a $k$-block model approximation of $W$, say $W[\hat{B}]$ for $\hat{B} \in \mathbb{R}^{k \times k}$, which minimizes the mean squared error,

$$
\mathbb{E}_{G \sim G_{n}(\rho W), \hat{B} \sim \mathcal{A}(G)}\left[\delta_{2}(\hat{B}, W)^{2}\right] .
$$

\subsection{Main Algorithm}

We use the same algorithm as Borgs et al. [15], described in Algorithm 1.

Notation for Algorithm 1 For $k, n \in \mathbb{N}$ with $k \leq n$, we say that $\pi:[n] \rightarrow[k]$ is a $k$-equipartition of $[n]$, if it partitions $[n]$ into $k$ classes such that is for every $i \in[n],|| \pi^{-1}(i)\left|-\frac{n}{k}\right|<1$. For a matrix $Q \in \mathbb{R}^{k \times k}$ and a matrix $A \in \mathbb{R}^{n \times n}$, we set $\operatorname{Score}(Q, \pi, A)=\|A\|_{2}^{2}-\left\|A-Q_{\pi}\right\|_{2}^{2}$, where $\pi$ ranges over all $k$-equipartitions of $[n],\left(Q_{\pi}\right)_{i, j}=Q_{\pi(i), \pi(j)}$ for all $i, j \in[n]$ and $\|A\|_{2}=\left(\frac{1}{n^{2}} \sum_{i, j=1}^{n} A_{i j}^{2}\right)^{\frac{1}{2}}$. Finally, we denote by $\mathcal{G}_{n}$ the space of undirected graphs on $n$ vertices and $\mathcal{G}_{n, d}$ the subset of graphs in $\mathcal{G}_{n}$ where the maximum degree is bounded by $d$.

We now describe the steps of the algorithm. The algorithm takes as input the privacy parameter $\varepsilon$, the graph $G$, a number $k$ of blocks, and a constant $\lambda \geq 1$ that will have to be chosen large enough to guarantee consistency of the algorithm.

Main Result Algorithm 1 is proven to be $\varepsilon$-node-DP at [15, Lemma 3]. Borgs et al gave upper bound on its worst-case mean squared error, $\mathbb{E}_{G \sim G_{n}(\rho W), \hat{B} \sim \mathcal{A}_{G}}\left[\delta_{2}(\hat{B}, W)^{2}\right]$. We state the improved bound here:

Theorem 3.1. Suppose

- $\frac{6 \log n}{n}<\rho \leq \frac{1}{\Lambda}, 8 \Lambda \leq \lambda$, and

- $\rho n \varepsilon / \log n \rightarrow+\infty, \varepsilon=O\left(k^{2} \log n / \lambda^{3}\right)$

Then the $\varepsilon$-node-DP Algorithm 1 from [15], $\mathcal{A}$, with input $\varepsilon, \lambda, k$ and $G$ outputs a pair $(\hat{\rho}, \hat{B}) \in$ $[0,1] \times[0,1]^{k \times k}$ with $\mathbb{E}_{G \sim G_{n}(\rho W), \hat{B} \sim \mathcal{A}_{G}}\left[\delta_{2}\left(\frac{1}{\hat{\rho}} \hat{B}, W\right)^{2}\right]$ of the order

$$
O\left(\mathbb{E}\left[\varepsilon_{k}^{(O)}(W)^{2}\right]+\mathbb{E}\left[\varepsilon_{n}(W)^{2}\right]+\lambda \frac{k-1}{n}\right)+O\left(\lambda\left(\frac{\log k}{\rho n}+\frac{k^{2}}{\rho n^{2}}\right)+\lambda^{2} \frac{k^{2} \log n}{n \varepsilon}+\frac{\lambda^{2}}{n^{2} \rho^{2} \varepsilon^{2}}\right) .
$$

The bound from Theorem 1 in [15] states that, under slightly different parameter assumptions, the mean squared error $\mathbb{E}_{G \sim G_{n}(\rho W), \hat{B} \sim \mathcal{A}_{G}}\left[\delta_{2}\left(\frac{1}{\hat{\rho}} \hat{B}, W\right)^{2}\right]$ is at most

$$
O\left(\mathbb{E}\left[\varepsilon_{k}^{(O)}(W)^{2}\right]+\mathbb{E}\left[\varepsilon_{n}(W)^{2}\right]\right)+O\left(\sqrt{\lambda^{2}\left(\frac{\log k}{\rho n}+\frac{k^{2}}{\rho n^{2}}\right)}\right)+O\left(\lambda^{2} \frac{k^{2} \log n}{n \varepsilon}+\frac{\lambda^{2}}{n^{2} \rho^{2} \varepsilon^{2}}\right) .
$$

The improvement therefore of our result lies on the $\varepsilon$-independent part of the bound. For convenience, we call this part of the bound the non-private part of the bound and the $\varepsilon$-dependent part, the private part of the bound. As we establish in the following subsection, the improvement of Theorem 3.1 on the non-private part is the optimal possible. 


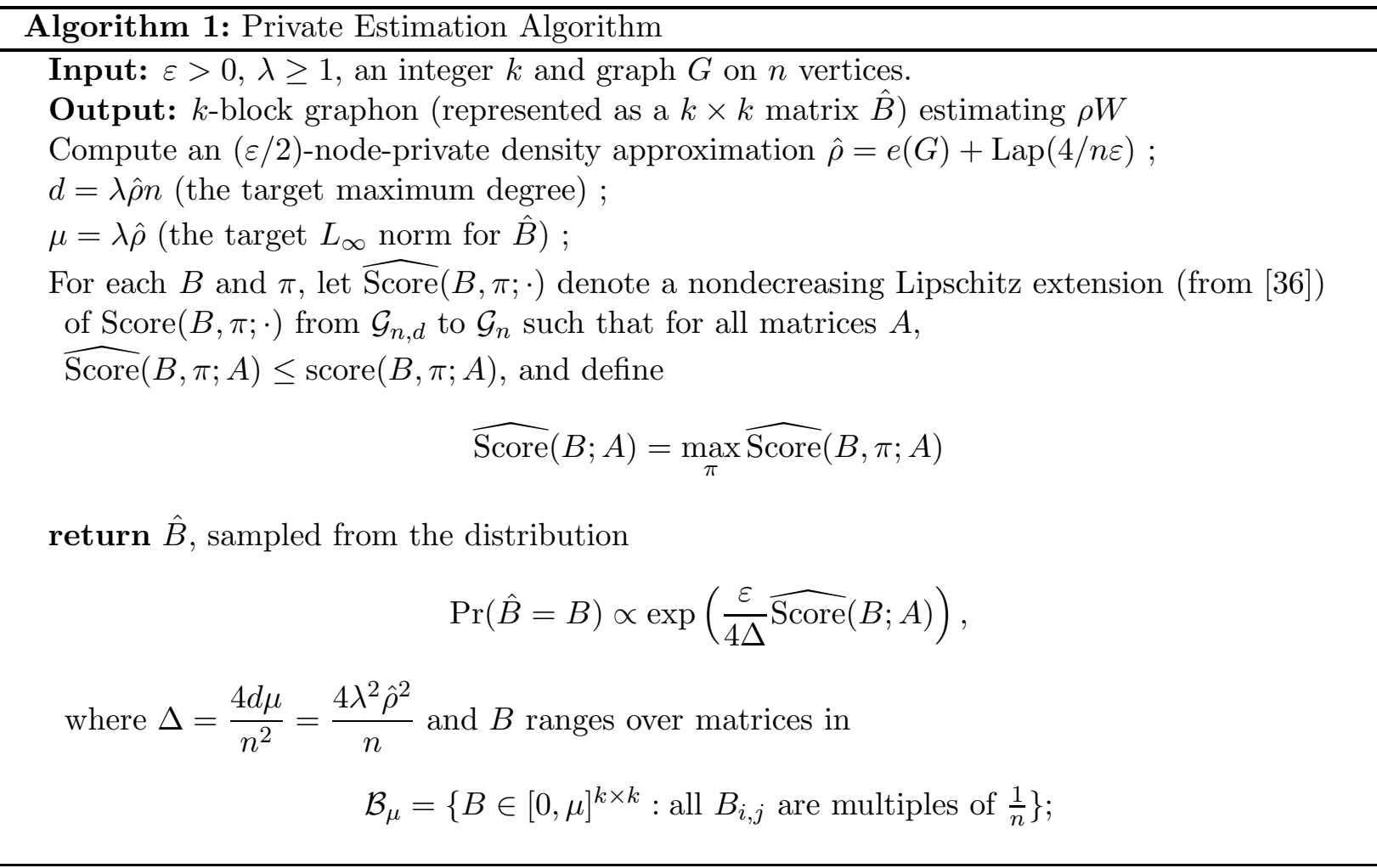

The $k$-block Estimation Rate In this subsection we focus on the case $W$ is a $k$-block graphon and establish that the improvement of Theorem 1 on the non-private part of the bound is optimal in the following sense. For some $k \geq 1$, assume that $W \in \mathcal{W}[k]$ with $\|W\|_{\infty} \leq \Lambda$, that is $W=W[B]$ for some $B \in[0, \Lambda]^{k \times k}$. Restricting ourselves to the specified subset of graphons we consider the minimax rate,

$$
\min _{\mathcal{A} \varepsilon-\text { node-DP } W \in \mathcal{W}[k],\|W\|_{\infty} \leq \Lambda} \mathbb{E}_{G \sim G_{n}(\rho W)}\left[\delta_{2}\left(\mathcal{A}_{G}, W\right)^{2}\right] .
$$

which we denote by $R_{k}(\rho, \varepsilon, \Lambda, n)$.

If $k \geq 2$, Theorem 3 from [44] and (up-to-log $k$ factors) Proposition 3.4 of [37], establishes that this rate, under no differential-privacy constraint (a case corresponding to $\varepsilon$ "equal to" $+\infty$ for our purposes), behaves like

$$
\Theta\left(\min \left\{\Lambda^{2} \sqrt{\frac{k}{n}}+\Lambda\left(\frac{\log k}{\rho n}+\frac{k^{2}}{\rho n^{2}}\right), \Lambda^{2}\right\}\right) .
$$

This result does not directly apply to our setting as we consider only finite $\varepsilon>0$. Note, though, that $\varepsilon$-node-DP is an increasing property, as if an algorithm is $\varepsilon$-node-DP, it is also $\varepsilon^{\prime}$-node-DP for any $\varepsilon^{\prime}>\varepsilon$. Therefore $R_{k}(\rho, \varepsilon, \Lambda, n)$ is a non-increasing function of $\varepsilon$, as increasing $\varepsilon$ only can shrink the feasible sets of estimators. Hence, the result from [37] provides a lower bound for the rate $R_{k}(\rho, \varepsilon, \Lambda, n)$. Combined with Theorem 3.1 we obtain a tight characterization of the nonprivate part of the rate $R_{k}(\rho, \varepsilon, \Lambda, n)$, and establish that Algorithm 1 from [15] obtains the optimal non-private part of the rate. 
Corollary 3.2. Suppose $k \geq 2$. Under the assumptions of Theorem 3.1 and the additional assumption $\rho n \geq k-2$,

$$
R_{k}(\rho, \varepsilon, \Lambda, n)=\Omega\left(\min \left\{\Lambda^{2} \sqrt{\frac{k}{n}}+\Lambda\left(\frac{\log k}{\rho n}+\frac{k^{2}}{\rho n^{2}}\right), \Lambda^{2}\right\}\right)
$$

and

$$
R_{k}(\rho, \varepsilon, \Lambda, n)=O\left(\Lambda^{2} \sqrt{\frac{k}{n}}+\Lambda\left(\frac{\log k}{\rho n}+\frac{k^{2}}{\rho n^{2}}\right)\right)+O\left(\Lambda^{2} \frac{k^{2} \log n}{n \varepsilon}+\frac{\Lambda^{2}}{n^{2} \rho^{2} \varepsilon^{2}}\right),
$$

where the upper bound is achieved by Algorithm 1 from [15].

A Lower Bound on the Private Part In this subsection we establish for $k \geq 2$ a lower bound on the private part of the rate. We establish that the term of order $\frac{\Lambda^{2}}{n^{2} \rho^{2} \varepsilon^{2}}$ appearing in the upper bound of Theorem 1 is necessary, up to the dependence on $\rho, \Lambda$. For the lower bound we focus on $k$-block graphons $W=W[B]$ with potentially slightly-unequal sizes, we do not require them to be normalized, and we set $\rho=\Lambda=1$. Specifically, let $\tilde{\mathcal{W}}[k]$ be the set of all graphons $W$ for which $\|W\|_{\infty} \leq 1$ and for some $A \in \mathbb{R}_{\geq 0}^{k \times k}$ and some $\mathcal{P}_{k}=\left(I_{1}, \ldots, I_{k}\right)$ partition of $[0,1]$ into adjacent intervals of (potentially different) lengths in $\left[\frac{1}{4 k}, \frac{4}{k}\right], W$ is the step function which equals $A_{i j}$ on $I_{i} \times I_{j}$, for every $i, j \in[k]$. Let also

$$
\tilde{R}_{k}(\varepsilon, n)=\min _{\mathcal{A} \varepsilon-\text { node-DP }} \max _{W \in \tilde{\mathcal{W}}[k]} \mathbb{E}_{G \sim G_{n}(W), \hat{B} \sim \mathcal{A}_{G}}\left[\delta_{2}(\hat{B}, W)^{2}\right] .
$$

Theorem 3.3. Suppose $k \geq 2$. Then

$$
\tilde{R}_{k}(\varepsilon, n)=\Omega\left(\frac{1}{n^{2} \varepsilon^{2}}\right)
$$

\section{Private Estimation of Erdos Renyi Graphs (1-Block Graphons)}

This section is devoted to the study of the privately estimating $k$-block graphons in the special case $k=1$. Since for $k=1$ the graphon corresponds to a constant function, we deal with the fundamental question of estimating privately the parameter of an Erdos-Renyi random graph model. Note that since the graphon is constant, to make the estimation task non-trivial we do not adopt the assumption that the graphon is normalized. Furthermore, using the notation of the previous section for reasons of simplicity we focus on the case $\rho$ is known to the analyst and $\Lambda=1$.

Using such a graphon $W$, we conclude that for some $p_{0} \in[0,1] W(x, y)=p_{0}$ for every $x, y \in[0,1]$ and the analyst's observes simply a sample from an Erdos Renyi random graph with $n$ vertices and parameter $p:=\rho \cdot p_{0} \leq \rho$. Multiplying the rate by the known $\rho$, the goal becomes to estimate $p$ using an $\varepsilon$-differentially private algorithm. In agreement with the non-private behavior where the estimation rate is provably much smaller when $k=1$ compared to $k>1$ (see Sec. 3.2 in [37] for details), we reveal a similar behavior in the case of private estimation. In particular, based on Theorem 3.3 for $k>1$ and $\Lambda=1$ the rate of interest is

$$
\Omega\left(\frac{1}{n^{2} \varepsilon^{2}}\right)
$$


Here we establish that the $\varepsilon$-dependent part of the rate for $k=1$ drops to

$$
O\left(\frac{\log n}{n^{3} \varepsilon^{2}}\right)
$$

\subsection{A New Algorithm for Density Estimation in Erdos Renyi Random Graphs}

The rate we want to find is for $\rho \in[0,1]$,

$$
R(\rho, \varepsilon, n)=\min _{\mathcal{A}} \max _{\varepsilon-\text { node-DP }} \mathbb{E}_{G \in[0, \rho]}\left[G_{n, p}\left[(A(G)-p)^{2}\right] .\right.
$$

A standard $\varepsilon$-node-DP algorithm for this task is the addition of appropriate Laplace noise to the edge density of the graph $G$ (Lemma 10 of [15]). The global sensitivity (Definition 2 in [15]) of the edge density with respect to the node-distance can be easily proven to be of the order $\Theta\left(\frac{1}{n}\right)$. In particular it is upper bounded by $\frac{4}{n}$, as if $G, G^{\prime} \in \mathbb{G}_{n}$,

$$
\left|e(G)-e\left(G^{\prime}\right)\right| \leq \frac{4}{n} d_{v}\left(G, G^{\prime}\right) .
$$

Therefore, using Lemma 10 of [15], the addition of $\operatorname{Lap}\left(\frac{4}{n \varepsilon}\right)$ noise to the edge density provides an $\varepsilon$-node-DP estimator. This estimator allows us to conclude the following Lemma.

Lemma 4.1. For any $\rho, \varepsilon>0$,

$$
R(\rho, \varepsilon, n)=O\left(\frac{\rho}{n^{2}}+\frac{1}{n^{2} \varepsilon^{2}}\right)
$$

As we establish in Theorem 4.3 the upper bound of Lemma 10 is, potentially surprisingly, not tight. A weakness of the proposed algorithm is that it computes an estimator based on the global sensitivity of the edge density over all pairs of undirected graphs of $n$ vertices and on the other hand applies it only to graphs coming from Erdos-Renyi models. To reveal more the potential weakness of the estimator, let us consider a pair of node-neighbors $G, G^{\prime}$, that is $d_{v}\left(G, G^{\prime}\right)=1$, where the difference $e(G)-e\left(G^{\prime}\right)$ is of the order $\frac{1}{n}$. It is easy to check that the difference can become of this order only if the degree of the rewired vertex had $o(n)$ degree in $G$ and $\Theta(n)$ degree in $G^{\prime}$ or vice versa. Since the degree of every other vertex changes by at most 1 , the rewired vertex in $G$ or $G^{\prime}$ has either very high degree or very low degree compared to the average degree in $G$ or $G^{\prime}$. Such a non-homogenuous property of the degree distribution appears, though, only with a negligible probability under any Erdos-Renyi model. This line of thought suggests that there could possibly be some "homogeneity" set, $\mathcal{H}$, for which any graph sampled from Erdos Renyi model belongs to with probability $1-o(1)$ and the sensitivity of the edge density on pairs of graphs from $\mathcal{H}$ is much lower than $\frac{1}{n}$.

Unfortunately the existence of such a set can be proven to be non-true for the following reason. The empty graph $G_{0}$ (which appears almost surely for the Erdos Renyi graph with $p=0$ ) and the complete graph $G_{1}$ (which appears almost surely for the Erdos Renyi random graph with $p=1$ ) should be included in such "homogeneity" set and furthermore

$$
\frac{e\left(G_{1}\right)-e\left(G_{0}\right)}{d_{v}\left(G_{0}, G_{1}\right)}=\frac{1}{n-1}=\Theta\left(\frac{1}{n}\right)
$$


We establish, though, that this is essentially the only "extreme" case and such an "homogeneity" set $\mathcal{H}$ exists, in the following sense. There exist a set $\mathcal{H}$ which contains any Erdos Renyi graph with probability $1-o(1)$, that is

$$
\min _{p \in[0,1]} \mathbb{P}_{G \sim G_{n, p}}(G \in \mathcal{H})=1-o(1),
$$

and furthermore from any $G, G^{\prime} \in \mathcal{H}$ either

$$
d_{v}\left(G, G^{\prime}\right)>n / 4
$$

or

$$
\frac{\left|e(G)-e\left(G^{\prime}\right)\right|}{d_{v}\left(G, G^{\prime}\right)}=O\left(\frac{\sqrt{\log n}}{n^{3 / 2}}\right) .
$$

This $\sqrt{n}$-improvement on the edge density sensitivity on $\mathcal{H}$ allows us to establish the existence of an $\varepsilon / 2$-node-DP algorithm which is defined on graphs in $\mathcal{H}$ and has mean squared error of the order $O\left(\frac{\log n}{n^{3} \varepsilon^{2}}\right)$. Notice that the order is much lower than the performance of the addition of Laplace noise (Lemma 10). Next we establish a general extension result (Theorem 5.1) which allows us to extend the $\varepsilon / 2$-node-DP algorithm defined on $\mathcal{H}$ to an $\varepsilon$-node-DP on the whole space of undirected graphs with $n$ nodes. The extension has the crucial property that it outputs the same probability distributions with the original algorithm when the input belongs in $\mathcal{H}$. The extension result applies generally to any $\varepsilon$-differentially private algorithm which takes values in an arbitrary metric space and outputs probability distributions of any measurable space. Since such a result could be of independent interest we devote Section 5 solely for its presentation.

Using the extented algorithm we establish the following results for graphs sampled from the Erdos Renyi random graph $G_{n, p}$ and the uniform graph $G(n, m)$. Notice that for the $G_{n, p}$ model there exists an additional non-private term $\frac{\rho}{n^{2}}$. This appears only in the Erdos-Renyi case and not in the uniform model as it comes from the vanishing but non-zero variance term of the edge density in the Erdos Renyi model.

Proposition 4.2 (The $G(n, m)$ case). Let $\varepsilon, \rho \in(0,1)$ be functions of $n$ such that $\varepsilon n / \log n \rightarrow+\infty$. There is an $\varepsilon$-node-DP algorithm $A$ such that, for all $m<\rho\left(\begin{array}{l}n \\ 2\end{array}\right)$,

$$
\underset{G \sim G(n, m)}{\mathbb{E}}\left|A(G)-\frac{m}{\left(\begin{array}{c}
n \\
2
\end{array}\right)}\right|^{2}=O\left(\max \left\{\rho, \frac{\log n}{n}\right\} \cdot \frac{\log n}{n^{3} \varepsilon^{2}}\right) .
$$

Theorem 4.3 (The Erdos-Renyi case). If $\varepsilon \in(0,1)$ with $\varepsilon n / \log n \rightarrow+\infty$, then

$$
R(\rho, \varepsilon, n)=O\left(\frac{\rho}{n^{2}}+\max \left\{\rho, \frac{\log n}{n}\right\} \frac{\log n}{n^{3} \varepsilon^{2}}\right) .
$$

\subsection{Lower bounds for $G(n, m)$}

In this subsection we dicuss the complementary question of lower bounds for the edge density estimation question in random graphs. We establish that when $\varepsilon$ in constant and the graph is generated by the uniform model $G(n, m)$, the bound implied by Proposition 4.2 is tight.

We establish this by first proving the following proposition on coupling of $G(n, m)$ models with varying $m$ which could be of independent interest. 
Proposition 4.4. Let $n$ be sufficiently large, and $k$ an arbitrary function of $n$ which is o( $\sqrt{n})$. Let $m=\frac{1}{2}\left(\begin{array}{l}n \\ 2\end{array}\right)-\frac{k}{2}$ Let $P=G(n, m)$ and $Q=G(n, m+k)$. There exists a coupling of $(G, H)$ of $P$ and $Q$ such that, with probability tending to one, one can obtain $G$ from $H$ by rewiring one vertex.

Using the proposition we establish the following lower bound.

Theorem 4.5. Let $\varepsilon>0$ be a constant positive number, $n \in \mathbb{N}, m=\frac{1}{2}\left(\begin{array}{l}n \\ 2\end{array}\right)-\frac{k}{2}$ and $k$ an arbitrary function of $n$ which is $o(\sqrt{n})$. Then there exists a $\beta=\beta(\varepsilon) \in(0,1)$ such that no $\varepsilon$-node $D P$ private algorithm can distinguish $G(n, m)$ from $G(n, m+k)$ with probability bigger than $\beta(\varepsilon)>0$. In particular, the upper bound of Proposition 4.2 is tight up-to-logarithmic terms for constant $\varepsilon$ and $\rho$.

\section{A General Extension Technique}

In this section we describe the general extension technique which allowed us to conclude the upper bound in Theorem 4.3. Since the technique applies generally to the extension of any $\varepsilon$-differentially private algorithm from any input metric space to any output measurable space, we present it here for the following general model.

The Model Let $n \in \mathbb{N}$ and $\varepsilon>0$. We assume that the analyst's objective is to estimate a certain quantity which takes values in some measurable space $(\Omega, \mathcal{F})$ from input data which take values in a metric space $(\mathcal{M}, d)$. The analyst is assumed to use for this task a randomized algorithm $\mathcal{A}$ which should be

(1) as highly accurate as possible for input data belonging in some hypothesis set $\mathcal{H} \subseteq \mathcal{M}$;

(2) $\varepsilon$-differentially private on the whole metric space of input data $(M, d)$.

In this section we state the following result. Consider an arbitrary $\varepsilon$-differentially private algorithm defined on input belonging in some set $\mathcal{H} \subset \mathcal{M}$. We show that it can be always extended to a $2 \varepsilon$-differentially private algorithm defined for arbitrary input data from $\mathcal{M}$ with the property that if the input data belongs in $\mathcal{H}$, the distribution of output values is the same with the original algorithm. We state formally the result.

Proposition 5.1 ("Extending Private Algorithms at $\varepsilon$-cost"). Let $\hat{\mathcal{A}}$ be an $\varepsilon$-differentially private algorithm designed for input from $\mathcal{H} \subseteq \mathcal{M}$. Then there exists a randomized algorithm $\mathcal{A}$ defined on the whole input space $\mathcal{M}$ which is $2 \varepsilon$-differentially private and satisfies that for every $D \in \mathcal{H}$, $\mathcal{A}(D) \stackrel{d}{=} \hat{\mathcal{A}}(D)$. 


\section{Proofs for Section 2}

Definitions and Notation For $A, B \in \mathbb{R}^{n \times n}$ and $1 \leq p<\infty$ we use the normalised $p$-norms,

$$
\|A\|_{p}=\left(\frac{1}{n^{2}} \sum_{i, j=1}^{n} A_{i j}^{p}\right)^{\frac{1}{p}}
$$

and the normalised inner product

$$
\langle A, B\rangle=\frac{1}{n^{2}} \sum_{i, j=1}^{n} A_{i j} B_{i j} .
$$

For $k, n \in \mathbb{N}$ with $k \leq n$, we say that $\pi:[n] \rightarrow[k]$ is a $k$-equipartition of $[n]$, if it partitions $[n]$ into $k$ classes all of which have size as close to $\frac{n}{k}$ as possible, that is for every $i \in[n],|| \pi^{-1}(i)\left|-\frac{n}{k}\right|<1$. For $Q \in \mathbb{R}^{k \times k}$ and $\pi$ a $k$-equipartition of $[n]$ we set $Q_{\pi}$ the block matrix given by $\left(Q_{\pi}\right)_{i j}=Q_{\pi(i) \pi(j)}$. Furthermore, if $\pi$ a $k$-equipartition of $[n]$ and $B \in \mathbb{R}^{n \times n}$ we let $B(\pi) \in \mathbb{R}^{k \times k}$ be the matrix with entries

$$
B(\pi)_{i j}=\frac{1}{\left|\pi^{-1}(i)\right|\left|\pi^{-1}(j)\right|} \sum_{l \in \pi^{-1}(i), m \in \pi^{-1}(j)} B_{l, m} .
$$

Notice that clearly $B(\pi)=\operatorname{argmin}_{B \in \mathbb{R}^{k \times k}}\left\|B_{\pi}-B\right\|_{2}$ and $\left\|B(\pi)_{\pi}-B\right\|_{2}=\min _{B \in \mathbb{R}^{k \times k}}\left\|B_{\pi}-B\right\|$.

We define the score function defined in [15]; for a matrix $Q \in \mathbb{R}^{k \times k}$ and a matrix $A \in \mathbb{R}^{n \times n}$ we set $\operatorname{Score}(Q, A)=\max _{\pi}\left(\|A\|_{2}^{2}-\left\|A-Q_{\pi}\right\|_{2}^{2}\right)$, where $\pi$ ranges over all $k$-equipartitions of $[n]$. For $r \in[0,1]$ and $k, n \in \mathbb{N}$ we set

$$
\mathcal{B}_{r}=\left\{B \in[0, r]^{k \times k} \mid n B_{i j} \in \mathbb{Z}, \text { for all } i, j \in[k]\right\} .
$$

For every $B \in[0, r]^{n \times n}$ and $\pi k$-equipartition of $[n]$, let $B(\pi, n) \in \mathcal{B}_{r}$ be the matrix with entries

$$
B(n, \pi)_{i j}=\frac{\left\lfloor n(B(\pi))_{i j}\right\rfloor}{n} .
$$

For some symmetric $Q \in[0,1]^{k \times k}$ such that $Q_{i i}=0$ for all $i=1,2, \ldots, n$ we say that a symmetric matrix $A \in \mathbb{R}^{k \times k}$ is distributed according to $\operatorname{Bern}_{0}(Q)$ if for all $i \leq j, A_{i, j}$ follows an independent Bernoulli with parameter $Q_{i, j}$.

Key Lemmata For this subsection we define between two matrices $B_{1} \in \mathbb{R}^{k \times k}$ and $B_{2} \in \mathbb{R}^{n \times n}$ the distance

$$
\hat{\delta}_{2}\left(B_{1}, B_{2}\right)=\min _{\pi}\left\|\left(B_{1}\right)_{\pi}-B_{2}\right\|_{2}
$$

where $\pi:[k] \rightarrow[n]$ ranges over all equipartitions of $[n]$ into $k$ classes. Furthermore for $Q \in \mathbb{R}^{n \times n}$ let $\hat{\varepsilon}_{k}^{(O)}(Q)=\min _{\pi, B \in \mathbb{R}^{k \times k}}\left\|B_{\pi}-Q\right\|_{2}=\min _{\pi}\left\|Q(\pi)_{\pi}-Q\right\|_{2}$.

Now, the essential improvement on the analysis of [15] is coming from improving Proposition 1 of [15] to the following proposition. 
Proposition 6.1. Let $r \in\left[\frac{1}{n}, 1\right], Q \in[0, r]^{n \times n}$ be a symmetric matrix with vanishing diagonal and $A \sim \operatorname{Bern}_{0}(Q)$. For $\hat{B} \in \mathcal{B}_{r}$ and $\nu \geq 0$ conditional on an event such that

$$
\mathcal{E} \subseteq\left\{\operatorname{Score}(\hat{B}, A) \geq \max _{B \in \mathcal{B}_{r}}[\operatorname{Score}(B, A)]-\nu^{2}\right\}
$$

the following holds

$$
\mathbb{E}\left[\hat{\delta}_{2}(\hat{B}, Q)^{2} \mid \mathcal{E}\right] \leq O\left(\hat{\varepsilon}_{k}^{(O)}(Q)^{2}+\nu^{2}+r\left(\frac{\log k}{n}+\frac{k^{2}}{n^{2}}\right)\right) .
$$

To prove Proposition 6.1 we need first two lemmata which can be established almost compeltely by the proof techniques of [37] . Despite that we fully establish them here for reader's convenience, using only one lemma from [37].

Lemma 6.2. Under the assumptions of Proposition (6.1) for every $\pi$ k-equipartition of $[n]$,

$$
\mathbb{E}\left[\sup _{\pi}\left(\left\langle Q-Q(\pi)_{\pi}, Q-A\right\rangle-\frac{1}{16}\left\|Q(\pi)_{\pi}-Q\right\|_{2}^{2}\right)\right] \leq O\left(r \frac{\log k}{n}\right)
$$

and

$$
\mathbb{E}\left[\sup _{\pi}\left(\left\langle Q(n, \pi)_{\pi}-Q, Q-A\right\rangle-\frac{1}{16}\left\|Q(n, \pi)_{\pi}-Q\right\|_{2}^{2}\right)\right] \leq O\left(r \frac{\log k}{n}\right)
$$

Proof. We establish only (6.3) as (6.4) follows similarly.

Recall first Bernstein's inequality which we state for reader's convenience. Let $X_{1}, \ldots, X_{N}$ independent zero-mean random variables. Suppose $\left|X_{i}\right| \leq M$ almost surely for all $i=1,2, \ldots, n$. Then for any $t>0$,

$$
\mathbb{P}\left[\sum_{i=1}^{N} X_{i} \geq \sqrt{2 t \sum_{i=1}^{N} \mathbb{E}\left[X_{i}^{2}\right]}+\frac{2 M}{3} t\right] \leq \exp (-t) .
$$

Now notice that for any $\pi$,

$$
\left\langle Q-Q(\pi)_{\pi}, Q-A\right\rangle=\frac{2}{n^{2}} \sum_{i<j}\left(Q-Q(\pi)_{\pi}\right)_{i j}\left(Q_{i j}-A_{i j}\right) .
$$

Furthermore for each $i<j,\left|\left(Q-Q(\pi)_{\pi}\right)_{i j}\right| \leq r$ and $\operatorname{Var}\left(A_{i, j}\right)=\operatorname{Var}\left(Q_{i, j}-A_{i, j}\right) \leq\|Q\|_{\infty} \leq r$. Therefore by Bernstein we conclude that for any $\pi$

$$
\mathbb{P}\left[\left\langle Q-Q(\pi)_{\pi}, Q-A\right\rangle \geq \frac{2}{n}\left\|Q-Q(\pi)_{\pi}\right\|_{2} \sqrt{r t}+\frac{4}{3 n^{2}} r t\right) \leq \exp (-t),
$$

for any $t>0$. Now taking a union bound over all $\pi$, which are at most $k^{n}=\exp (k \log n)$, we conclude that

$$
\mathbb{P}\left[\exists \pi:\left\langle Q-Q(\pi)_{\pi}, Q-A\right\rangle \geq \frac{2}{n}\left\|Q-Q(\pi)_{\pi}\right\|_{2} \sqrt{r(t+k \log n)}+\frac{4}{3 n^{2}} r(t+k \log n)\right) \leq \exp (-t),
$$

for any $t>0$. Using the elementary $2 u v \leq u^{2}+v^{2}$ we conclude

$$
\mathbb{P}\left[\exists \pi:\left\langle Q-Q(\pi)_{\pi}, Q-A\right\rangle-\frac{1}{16}\left\|Q-Q(\pi)_{\pi}\right\|_{2}^{2} \geq \frac{52}{3 n^{2}} r(t+k \log n)\right) \leq \exp (-t),
$$


for any $t>0$, or for $C=\frac{52}{3}$,

$$
\mathbb{P}\left[\sup _{\pi}\left(\left\langle Q-Q(\pi)_{\pi}, Q-A\right\rangle-\frac{1}{16}\left\|Q-Q(\pi)_{\pi}\right\|_{2}^{2}\right) \geq \frac{C}{n^{2}} r(t+n \log k)\right) \leq \exp (-t),
$$

for any $t>0$. Integration with respect to $t$ implies the statement of the Lemma.

Lemma 6.3. Under the assumptions of Proposition (6.1),

$$
\mathbb{E}\left[\sup _{C, \pi}\left(\left\langle C_{\pi}, Q-A\right\rangle-\frac{1}{16}\|C\|_{2}^{2}\right)\right] \leq O\left(r\left(\frac{\log k}{n}+\frac{k^{2}}{n^{2}}\right)\right)
$$

where the optimization is over all $C \in \mathcal{B}_{r}$ and $\pi k$-equipartitions of $[n]$.

Proof. We care to control the quantity $\sup _{C, \pi}\left(\left\langle C_{\pi}, Q-A\right\rangle-\frac{1}{16}\|C\|_{2}^{2}\right)$. The quantity of interest equals

$$
\sup _{R \geq 0, \pi} \sup _{C \in \mathcal{B}_{r},\|C\|_{2}=R}\left(\left\langle C_{\pi}, Q-A\right\rangle-\frac{1}{16} R^{2}\right) \leq \sup _{R \geq 0, \pi}\left(\sup _{C \in[0,2 r]^{k \times k}:\|C\|_{2} \leq R}\left(\left\langle C_{\pi}, Q-A\right\rangle\right)-\frac{1}{16} R^{2}\right) .
$$

Now fix $\pi, R$, We set $A(\pi, R)=\left\{C_{\pi}: C \in[0,2 r]^{k \times k}:\|C\|_{2} \leq R\right\}$ and consider $D(R)_{\pi} \in A(\pi, R)$ with

$$
\left\langle D(R)_{\pi}, Q-A\right\rangle=\sup _{C \in A(\pi, R)}\left\langle C_{\pi}, Q-A\right\rangle .
$$

What we care to bound is

$$
\sup _{R \geq 0, \pi}\left(\left\langle D(R)_{\pi}, Q-A\right\rangle-\frac{1}{16} R^{2}\right)
$$

We split two cases. If $\left\|D(R)_{\pi}\right\|_{2} \leq \frac{2 r}{n}$ we have by Cauchy-Schwarz that

$$
\left\langle D(R)_{\pi}, Q-A\right\rangle \leq\left\|D(R)_{\pi}\right\|_{2}\|Q-A\|_{2} \leq \frac{2 r}{n},
$$

since $\|Q-A\|_{\infty} \leq 1$, and therefore

$$
\sup _{R \geq 0, \pi}\left(\left\langle D(R)_{\pi}, Q-A\right\rangle-\frac{1}{16} R^{2}\right) \leq \frac{2 r}{n} .
$$

If $\left\|D(R)_{\pi}\right\|_{2} \geq \frac{2 r}{n}$ we use Lemma 4.1 of [37]. In that lemma the authors construct a subset $\mathcal{C}^{*}(\pi) \subset\left\{C_{\pi}: C \in[0,2 r]^{k \times k}\right\}$ with the following two properties,

(1) $\log \left|\mathcal{C}^{*}(\pi)\right|=O\left(k^{2}+\log \log n\right)$

(2) For any $R>0$, if $\left\|D(R)_{\pi}\right\|_{2} \geq \frac{2 r}{n}$ then there exists $V_{\pi} \in \mathcal{C}^{*}(\pi)$ such that $\|D(R)-V\|_{2} \leq$ $\frac{\|D(R)\|_{2}}{4}$ and $\|D(R)-V\|_{\infty} \leq r$. 
Hence if $\left\|D(R)_{\pi}\right\|_{2} \geq \frac{2 r}{n}$ then for the $V$ satisfying (2) we have the following two properties. First $\|V\|_{2} \leq \frac{5}{4}\|D(R)\|_{2}<2 R$. Furthermore clearly $2\left(D(R)_{\pi}-V_{\pi}\right) \in A_{\pi, R}$. Therefore by the definition of $D$ and the case we consider we have $\left\langle 2\left(D(R)_{\pi}-V_{\pi}\right), Q-A\right\rangle \leq\left\langle D(R)_{\pi}, Q-A\right\rangle$ or

$$
\left\langle D(R)_{\pi}, Q-A\right\rangle \leq 2\left\langle V_{\pi}, Q-A\right\rangle .
$$

We conclude combining the above that

$$
\left\langle D(R)_{\pi}, Q-A\right\rangle \leq 2 \sup _{C \in \mathcal{C}^{*}(\pi) \cap\left\{C:\|C\|_{2} \leq 2 R\right\}}\left\langle C_{\pi}, Q-A\right\rangle .
$$

Therefore it suffices to bound the expectation of the quantity

$$
\sup _{R \geq 0, \pi}\left(2 \sup _{C \in \mathcal{C}^{*}(\pi) \cap\left\{C:\|C\|_{2} \leq 2 R\right\}}\left(\left\langle C_{\pi}, Q-A\right\rangle\right)-\frac{1}{16} R^{2}\right)
$$

which is at most

$$
2 \sup _{R \geq 0, \pi, C \in \mathcal{C}^{*}(\pi) \cap\left\{C:\|C\|_{2} \leq 2 R\right\}}\left(\left\langle C_{\pi}, Q-A\right\rangle-\frac{1}{128}\|C\|_{2}^{2}\right)
$$

which equals

$$
2 \sup _{\pi, C \in \mathcal{C}^{*}(\pi)}\left(\left\langle C_{\pi}, Q-A\right\rangle-\frac{1}{128}\|C\|_{2}^{2}\right)
$$

Combining both cases we conclude that in complete generality

$$
\sup _{R>0, \pi}\left(2 \sup _{C \in \mathcal{C}^{*}(\pi) \cap\left\{C:\|C\|_{2} \leq R\right\}}\left(\left\langle C_{\pi}, Q-A\right\rangle\right)-\frac{1}{16} R^{2}\right) \leq \frac{2 r}{n}+2 \sup _{\pi, C \in \mathcal{C}^{*}(\pi)}\left(\left\langle C_{\pi}, Q-A\right\rangle-\frac{1}{128}\|C\|_{2}^{2}\right) .
$$

We focus on the second term. As in the proof of Lemma 6.2 we have that for any $C \in[0,2 r]^{k \times k}$, $\pi$ k-equipartition of $[n]$,

$$
\mathbb{P}\left[\left\langle C_{\pi}, Q-A\right\rangle \geq \frac{2}{n}\left\|C_{\pi}\right\|_{2} \sqrt{2 r t}+\frac{8}{3 n^{2}} r t\right) \leq \exp (-t),
$$

for any $t>0$. Using that $\left\|C_{\pi}\right\|_{2}=\|C\|_{2}$ and the elementary $2 u v \leq u^{2}+v^{2}$ we conclude that for some $c_{0}>0$ and any $C \in[0,2 r]^{k \times k}, \pi$ k-equipartition of $[n]$,

$$
\mathbb{P}\left[\left\langle C_{\pi}, Q-A\right\rangle-\frac{1}{128}\|C\|_{2}^{2} \geq \frac{c_{0}}{n^{2}} r t\right) \leq \exp (-t),
$$

for any $t>0$. Hence using a union bound over all partitions $\pi$, which are at most $k^{n}$ and $C \in \mathcal{C}^{*}(\pi)$ which based on property (1) are $\exp \left(O\left(k^{2}+\log \log n\right)\right)=\exp \left(O\left(k^{2}+n \log k\right)\right)$ we derive that for some $c_{1}>0$

$$
\mathbb{P}\left[\sup _{\pi, C \in \mathcal{C}^{*}(\pi)}\left\langle C_{\pi}, Q-A\right\rangle-\frac{1}{128}\|C\|_{2}^{2} \geq \frac{6 c_{1}}{n^{2}} r\left(t+k \log n+k^{2}\right)\right) \leq \exp (-t),
$$

for any $t>0$. Integrating over $t>0$ yields that

$$
\mathbb{E}\left[\sup _{C \in \mathcal{C}^{*}(\pi), \pi}\left(\left\langle C_{\pi}, Q-A\right\rangle-\frac{1}{128}\|C\|_{2}^{2}\right)\right] \leq O\left(r\left(\frac{\log k}{n}+\frac{k^{2}}{n^{2}}\right)\right)
$$


Therefore from (6.7)

$$
\mathbb{E}\left[\sup _{C, \pi}\left(\left\langle C_{\pi}, Q-A\right\rangle-\frac{1}{128}\|C\|_{2}^{2}\right)\right] \leq O\left(\frac{r}{n}\right)+O\left(r\left(\frac{\log k}{n}+\frac{k^{2}}{n^{2}}\right)\right)=O\left(r\left(\frac{\log k}{n}+\frac{k^{2}}{n^{2}}\right)\right),
$$

which completes the proof.

Proof of Proposition 6.1. For any $B \in \mathcal{B}_{r}$, $\operatorname{Score}(B, A)=\max _{\pi}\left(\|A\|_{2}^{2}-\left\|A-B_{\pi}\right\|_{2}^{2}\right)$, where $\pi$ ranges over all equipartitions $\pi:[n] \rightarrow[k]$. Therefore, the equation (6.1) implies that conditioning on $\mathcal{E}$ we have

$$
\min _{\pi}\left\|\hat{B}_{\pi}-A\right\|_{2}^{2} \leq \min _{B \in \mathcal{B}_{r}, \pi}\left\|B_{\pi}-A\right\|_{2}^{2}+\nu^{2},
$$

By calling $\hat{\pi}$ the optimal permutation on the left hand side we conclude

$$
\left\|\hat{B}_{\hat{\pi}}-A\right\|_{2}^{2} \leq \min _{B \in \mathcal{B}_{r}, \pi}\left\|B_{\pi}-A\right\|_{2}^{2}+\nu^{2},
$$

Now recall that $\|Q\|_{\infty} \leq r$ and $\mathcal{B}_{r}$ is the set of matrices with elements which are arbitrary multiples of $\frac{1}{n}$ in $[0, r]$. In particular for any $k$-equipartition of $[n] \pi, Q(n, \pi) \in \mathcal{B}_{r}$. Since $\| Q(n, \pi)-$ $Q(\pi) \|_{\infty} \leq \frac{1}{n}$, using the elementary $(u+v)^{2} \leq 2\left(u^{2}+v^{2}\right)$ we have

$$
\left\|Q(n, \pi)_{\pi}-Q\right\|_{2}^{2} \leq 2\left\|Q(\pi)_{\pi}-Q\right\|_{2}^{2}+\frac{2}{n^{2}}
$$

which implies by the definition of $Q(\pi)$,

$$
\left\|Q(n, \pi)_{\pi}-Q\right\|_{2}^{2} \leq 2 \min _{B \in \mathbb{R}^{k \times k}, \pi}\left\|B_{\pi}-Q\right\|_{2}^{2}+\frac{2}{n^{2}}=2 \hat{\varepsilon}_{k}^{(O)}(Q)^{2}+\frac{2}{n^{2}} .
$$

Set $\tilde{\nu}^{2}=\nu^{2}+\frac{1}{n^{2}}$. Equation (6.11) since $Q(n, \pi) \in \mathcal{B}_{r}$ gives

$$
\left\|\hat{B}_{\hat{\pi}}-A\right\|_{2}^{2} \leq\left\|Q(n, \pi)_{\pi}-A\right\|_{2}^{2}+\nu^{2}
$$

almost surely conditional on $\mathcal{E}$, which now after adding and substracting $Q$ inside both the 2-norms and expanding them implies

$$
\left\|\hat{B}_{\hat{\pi}}-Q\right\|_{2}^{2} \leq\left\|Q(n, \pi)_{\pi}-Q\right\|_{2}^{2}+2\left\langle Q(n, \pi)_{\pi}-\hat{B}_{\hat{\pi}}, Q-A\right\rangle+\nu^{2} .
$$

almost surely conditional on $\mathcal{E}$. Adding and substracting $Q$ and $Q(\hat{\pi})_{\hat{\pi}}$ we have

$$
\left\|\hat{B}_{\hat{\pi}}-Q\right\|_{2}^{2} \leq\left\|Q(n, \pi)_{\pi}-Q\right\|_{2}^{2}+2\left\langle Q(n, \pi)_{\pi}-Q, Q-A\right\rangle+2\left\langle Q-Q(\hat{\pi})_{\hat{\pi}}, Q-A\right\rangle+2\left\langle(Q(\hat{\pi})-\hat{B})_{\hat{\pi}}, Q-A\right\rangle+\nu^{2}
$$

almost surely conditional on $\mathcal{E}$, and therefore $\mathbb{E}\left[\left\|\hat{B}_{\hat{\pi}}-Q\right\|_{2}^{2} \mid \mathcal{E}\right]$ is at most

$$
\left\|Q(n, \pi)_{\pi}-Q\right\|_{2}^{2}+2 \mathbb{E}\left[\left\langle Q(n, \pi)_{\pi}-Q, Q-A\right\rangle+\left\langle Q-Q(\hat{\pi})_{\hat{\pi}}, Q-A\right\rangle \mid \mathcal{E}\right]+2 \mathbb{E}\left[\left\langle(Q(\hat{\pi})-\hat{B})_{\hat{\pi}}, Q-A\right\rangle \mid \mathcal{E}\right]+\nu^{2}
$$


Bounding now the first two expected inner product terms according to Lemma 6.2 and the last according to Lemma 6.3 we obtain that the quantity $\mathbb{E}\left[\left\|\hat{B}_{\hat{\pi}}-Q\right\|_{2}^{2} \mid \mathcal{E}\right]$ is at most

$$
\begin{aligned}
& \frac{18}{16}\left\|Q(n, \pi)_{\pi}-Q\right\|_{2}^{2}+\frac{2}{16} \mathbb{E}\left[\left\|Q(\hat{\pi})_{\hat{\pi}}-Q\right\|_{2}^{2}+\left\|(Q(\hat{\pi})-\hat{B})_{\hat{\pi}}\right\|_{2}^{2} \mid \mathcal{E}\right]+O\left(r\left(\frac{\log k}{n}+\frac{k^{2}}{n^{2}}\right)\right)+\nu^{2} \\
& =\frac{9}{8}\left\|Q(n, \pi)_{\pi}-Q\right\|_{2}^{2}+\frac{1}{8} \mathbb{E}\left[\left\|Q(\hat{\pi})_{\hat{\pi}}-Q\right\|_{2}^{2}+\left\|(Q(\hat{\pi})-\hat{B})_{\hat{\pi}}\right\|_{2}^{2} \mid \mathcal{E}\right]+O\left(r\left(\frac{\log k}{n}+\frac{k^{2}}{n^{2}}\right)\right)+\nu^{2}
\end{aligned}
$$

Set $\tilde{\nu}^{2}=\nu^{2}+\frac{1}{n^{2}}$. From (6.12) we conclude

$\mathbb{E}\left[\left\|\hat{B}_{\hat{\pi}}-Q\right\|_{2}^{2} \mid \mathcal{E}\right] \leq \frac{1}{8} \mathbb{E}\left[\left\|Q(\hat{\pi})_{\hat{\pi}}-Q\right\|_{2}^{2}+\left\|(Q(\hat{\pi})-\hat{B})_{\hat{\pi}}\right\|_{2}^{2} \mid \mathcal{E}\right]+O\left(\hat{\varepsilon}_{k}^{(O)}(Q)^{2}+r\left(\frac{\log k}{n}+\frac{k^{2}}{n^{2}}\right)+\tilde{\nu}^{2}\right)$.

For any $k$-equipartition $\pi$, the matrix $Q(\pi)_{\pi}$ is the minimizer of the quantity $\left\|C_{\pi}-Q\right\|_{2}^{2}$ over all matrices $C \in \mathbb{R}^{k \times k}$. Therefore

$$
\left\|Q(\hat{\pi})_{\hat{\pi}}-Q\right\|_{2} \leq\left\|\hat{B}_{\hat{\pi}}-Q\right\|_{2}
$$

almost surely, and by triangle inequality also

$$
\left\|(Q(\hat{\pi})-\hat{B})_{\hat{\pi}}\right\|_{2} \leq\left\|\hat{B}_{\hat{\pi}}-Q\right\|_{2}+\left\|Q(\hat{\pi})_{\hat{\pi}}-Q\right\|_{2} \leq 2\left\|\hat{B}_{\hat{\pi}}-Q\right\|_{2},
$$

almost surely. Hence combining the last inequalities together we obtain

$$
\mathbb{E}\left[\left\|\hat{B}_{\hat{\pi}}-Q\right\|_{2}^{2} \mid \mathcal{E}\right] \leq \frac{5}{8} \mathbb{E}\left[\left\|\hat{B}_{\hat{\pi}}-Q\right\|_{2}^{2} \mid \mathcal{E}\right]+O\left(\hat{\varepsilon}_{k}^{(O)}(Q)^{2}+r\left(\frac{\log k}{n}+\frac{k^{2}}{n^{2}}\right)+\tilde{\nu}^{2}\right)
$$

or

$$
\mathbb{E}\left[\left\|\hat{B}_{\hat{\pi}}-Q\right\|_{2}^{2} \mid \mathcal{E}\right]=O\left(\hat{\varepsilon}_{k}^{(O)}(Q)^{2}+r\left(\frac{\log k}{n}+\frac{k^{2}}{n^{2}}\right)+\tilde{\nu}^{2}\right) .
$$

Finally notice that since $r \geq \frac{1}{n}$,

$$
\tilde{\nu}^{2}=\nu^{2}+\frac{1}{n^{2}}=O\left(\nu^{2}+r \frac{\log k}{n}\right)
$$

Plugging this in (6.14) we obtain

$$
\mathbb{E}\left[\left\|\hat{B}_{\hat{\pi}}-Q\right\|_{2}^{2} \mid \mathcal{E}\right]=O\left(\hat{\varepsilon}_{k}^{(O)}(Q)^{2}+r\left(\frac{\log k}{n}+\frac{k^{2}}{n^{2}}\right)+\nu^{2}\right) .
$$

This completes the proof of the Proposition 6.1

We need the following Lemma from [15].

Lemma 6.4. (Lemma 8 in [15]) Let $(\hat{\rho}, \hat{B})$ be the output of Algorithm 1 from [15] and $A$ the adjacency matrix of the observed graph $G$. Then under the assumptions of Theorem 1 the following properties hold with probability at least $1-2 \exp (-\Omega(n \rho \varepsilon))$; 
- $|e(G)-\hat{\rho}| \leq \frac{\rho}{4}$.

- If $d_{\max }(G) \leq \frac{\lambda \rho}{4}$ and $e(G) \geq \frac{\rho}{2}$, then

$$
\operatorname{Score}(\hat{B}, A) \geq \max _{B \in \mathcal{B}_{\lambda \hat{\rho}}}[\operatorname{Score}(B, A)]-O\left(\frac{\lambda^{2} \hat{\rho}^{2} k^{2} \log n}{n \varepsilon}\right) .
$$

We also need the following lemma.

Lemma 6.5. Let $B$ be $k \times k$ symmetric matrix with non negative entries and let $\pi$ be the standard equipartition of $[n]$ into $k$ classes. Then

$$
\left\|W\left[B_{\pi}\right]-W[B]\right\|_{2} \leq \sqrt{\frac{10(k-1)}{n}}\|B\|_{2} .
$$

Proof. If $k=1$ then $W\left[B_{\pi}\right]=W[B]$ and both the left and right hand side are zero.

If $k \geq 2$ we observe that $\sqrt{\frac{10(k-1)}{n}}\|B\|_{2}>\sqrt{\frac{4 k}{n}}\|B\|_{2}$ and the rest of the proof follows from Lemma 7 in [15].

Now we present a proof of Theorem 3.1,

Proof of Theorem 3.1. We first claim that with probability at least $1-n e^{-\Omega(\varepsilon \rho n)}-O\left(\frac{\Lambda}{n}\right)$ both conditions of the second part of Lemma 6.4, $d_{\max }(G) \leq \frac{\lambda \rho}{4}$ and $e(G) \geq \frac{\rho}{2}$, are satisfied. This follows since $\rho \log n \geq 6 n$ and $\Lambda \leq \lambda / 8$ by the Lemmata 13 and 12 from [15], respectively.

Consider the event $\mathcal{E}=\left\{\operatorname{Score}(\hat{B}, A) \geq \max _{B \in \mathcal{B}_{r}}[\operatorname{Score}(B, A)]-\nu^{2}\right\} \cap\left\{\frac{1}{10} \rho \leq \hat{\rho} \leq 10 \rho\right\}$ for our $A$ which follows $\operatorname{Bern}_{0}(Q)$ for $Q=\rho H_{n}(W)$, and parameters $r=10 \lambda \rho$ and $\nu=O\left(\lambda \hat{\rho} \sqrt{\frac{k^{2} \log n}{n \varepsilon}}\right)$. Using the second part of Lemma 8.1 we obtain that

$$
\mathbb{P}(\mathcal{E})=1-n e^{-\Omega(\varepsilon \rho n)}-O\left(\frac{\Lambda}{n}\right)=1-O\left(\frac{\Lambda}{n}\right),
$$

where for the last equality we used that $n \varepsilon \rho / \log n \rightarrow+\infty$. Hence using also that on $\mathcal{E},\|B\|_{2} \leq$ $\lambda \hat{\rho} \leq 10 \lambda \rho=r$. Therefore all the conditions of Proposition 6.1 are satisfied for the event $\mathcal{E}$.

Hence,

$$
\begin{aligned}
& \mathbb{E}\left[\hat{\delta}_{2}\left(\hat{B}, \rho H_{n}(W)\right)^{2}\right]=\mathbb{P}(\mathcal{E}) \mathbb{E}\left[\hat{\delta}_{2}\left(\hat{B}, \rho H_{n}(W)\right)^{2} \mid \mathcal{E}\right]+\mathbb{P}\left(\mathcal{E}^{c}\right) \mathbb{E}\left[\hat{\delta}_{2}\left(\hat{B}, \rho H_{n}(W)\right)^{2} \mid \mathcal{E}^{c}\right] \\
& \leq \mathbb{E}\left[\hat{\delta}_{2}\left(\hat{B}, \rho H_{n}(W)\right)^{2} \mid \mathcal{E}\right]+O\left(\frac{\Lambda}{n}\right) \mathbb{E}\left[\hat{\delta}_{2}\left(\hat{B}, \rho H_{n}(W)\right)^{2} \mid \mathcal{E}^{c}\right] \\
& \leq O\left(\mathbb{E}\left[\hat{\varepsilon}_{k}^{(O)}\left(\rho H_{n}(W)\right)^{2}\right]+\lambda \rho\left(\frac{\log k}{n}+\frac{k^{2}}{n^{2}}\right)+\lambda^{2} \rho^{2} \frac{k^{2} \log n}{n \varepsilon}\right)+O\left(\hat{\rho}^{2} \lambda^{2} \frac{\Lambda}{n}\right),
\end{aligned}
$$

where for the last inequality we used the crude bound $\hat{\delta}_{2}\left(\hat{B}, \rho H_{n}(W)\right) \leq O(\lambda \hat{\rho})$ on its complement $\mathcal{E}^{c}$ and Proposition 6.1 on $\mathcal{E}$. Note that Proposition 6.1 can be applied because $\rho$ is assumed to be bigger than $\frac{6 \log n}{n}$. Now since $\hat{\rho}$ is $e(G)$ with Laplace noise of parameter $O\left(\frac{1}{n \varepsilon}\right)$ we can 
easily conclude that $\hat{\rho}$ is stochastically dominated by the addition of a $\frac{1}{n^{2}} \operatorname{Binom}\left(\left(\begin{array}{l}n \\ 2\end{array}\right), \Lambda \rho\right)$ and an independent $\operatorname{Lap}\left(\frac{4}{n \varepsilon}\right)$. In particular

$$
\mathbb{E}\left[\hat{\rho}^{2}\right] \leq O\left(\Lambda^{2} \rho^{2}+\frac{\Lambda^{2}}{n^{2} \varepsilon^{2}}\right)=O\left(\Lambda^{2} \rho^{2}\right)
$$

or

$$
\mathbb{E}\left[\frac{\hat{\rho}^{2}}{\rho^{2}}\right]=O\left(\Lambda^{2}\right)
$$

Hence rescaling by $\rho^{2}$ we have

$$
\mathbb{E}\left[\hat{\delta}_{2}\left(\frac{1}{\rho} \hat{B}, H_{n}(W)\right)^{2}\right] \leq O\left(\mathbb{E}\left[\hat{\varepsilon}_{k}^{(O)}\left(H_{n}(W)\right)^{2}\right]+\lambda\left(\frac{\log k}{\rho n}+\frac{k^{2}}{\rho n^{2}}\right)+\lambda^{2} \frac{k^{2} \log n}{n \varepsilon}+\lambda^{2} \frac{\Lambda^{3}}{n}\right) .
$$

Using triangle inequality for the $\ell_{2}$ norm and the elementary $(u+v)^{2} \leq 2\left(u^{2}+v^{2}\right)$ we know that

$$
\mathbb{E}\left[\hat{\delta}_{2}\left(\frac{1}{\hat{\rho}} \hat{B}, H_{n}(W)\right)^{2}\right]=O\left(\mathbb{E}\left[\hat{\delta}_{2}\left(\frac{1}{\rho} \hat{B}, H_{n}(W)\right)^{2}\right]+\mathbb{E}\left[\left\|\frac{1}{\hat{\rho}} \hat{B}-\frac{1}{\rho} \hat{B}\right\|_{2}^{2}\right]\right) .
$$

Hence $\mathbb{E}\left[\hat{\delta}_{2}\left(\frac{1}{\hat{\rho}} \hat{B}, H_{n}(W)\right)^{2}\right]$ is at most of the order

$$
O\left(\mathbb{E}\left[\hat{\varepsilon}_{k}^{(O)}\left(H_{n}(W)\right)^{2}\right]+\lambda\left(\frac{\log k}{\rho n}+\frac{k^{2}}{\rho n^{2}}\right)+\lambda^{2} \frac{k^{2} \log n}{n \varepsilon}+\lambda^{2} \frac{\Lambda^{3}}{n}+\mathbb{E}\left[\|\hat{B}\|_{2}^{2}\left(\frac{1}{\hat{\rho}}-\frac{1}{\rho}\right)^{2}\right]\right) .
$$

or since $\varepsilon=O\left(k^{2} \log n / \lambda^{3}\right)=O\left(k^{2} \log n / \Lambda^{3}\right)$, at most of the order

$$
O\left(\mathbb{E}\left[\hat{\varepsilon}_{k}^{(O)}\left(H_{n}(W)\right)^{2}\right]+\lambda\left(\frac{\log k}{\rho n}+\frac{k^{2}}{\rho n^{2}}\right)+\lambda^{2} \frac{k^{2} \log n}{n \varepsilon}+\mathbb{E}\left[\|\hat{B}\|_{2}^{2}\left(\frac{1}{\hat{\rho}}-\frac{1}{\rho}\right)^{2}\right]\right) .
$$

Now we focus on the term $\mathbb{E}\left[\|\hat{B}\|_{2}^{2}\left(\frac{1}{\hat{\rho}}-\frac{1}{\rho}\right)^{2}\right]$. since $\hat{B} \in \mathcal{B}_{\lambda \hat{\rho}}$ we have that $\|\hat{B}\|_{2} \leq \lambda \hat{\rho}$ almost surely. Therefore,

$$
\mathbb{E}\left[\|\hat{B}\|_{2}^{2}\left(\frac{1}{\hat{\rho}}-\frac{1}{\rho}\right)^{2}\right] \leq \lambda^{2} \mathbb{E}\left[\left(1-\frac{\hat{\rho}}{\rho}\right)^{2}\right]=\frac{\lambda^{2}}{\rho^{2}} \mathbb{E}\left[(\rho-\hat{\rho})^{2}\right] .
$$

Using that $\hat{\rho}=e(G)+\operatorname{Lap}\left(\frac{4}{n \varepsilon}\right)$ we conclude

$$
\mathbb{E}\left[\|\hat{B}\|_{2}^{2}\left(\frac{1}{\hat{\rho}}-\frac{1}{\rho}\right)^{2}\right] \leq \frac{\lambda^{2}}{\rho^{2}}\left(\mathbb{E}\left[(\rho-e(G))^{2}\right]+O\left(\frac{1}{n^{2} \varepsilon^{2}}\right)\right) .
$$

Using Lemma 12 in [15] we have that $\mathbb{E}\left[(\rho-e(G))^{2}\right]=O\left(\frac{\rho^{2} \Lambda}{n}\right)$ and therefore

$$
\mathbb{E}\left[\|\hat{B}\|_{2}^{2}\left(\frac{1}{\hat{\rho}}-\frac{1}{\rho}\right)^{2}\right] \leq O\left(\frac{\lambda^{2} \Lambda}{n}+\frac{\lambda^{2}}{n^{2} \varepsilon^{2} \rho^{2}}\right) .
$$

Using that to (6.15) we obtain that $\mathbb{E}\left[\hat{\delta}_{2}\left(\frac{1}{\rho} \hat{B}, H_{n}(W)\right)^{2}\right]$ is at most of the order

$$
O\left(\mathbb{E}\left[\hat{\varepsilon}_{k}^{(O)}\left(H_{n}(W)\right)^{2}\right]+\lambda\left(\frac{\log k}{\rho n}+\frac{k^{2}}{\rho n^{2}}\right)+\lambda^{2} \frac{k^{2} \log n}{n \varepsilon}+\frac{\lambda^{2} \Lambda}{n}+\frac{\lambda^{2}}{n^{2} \varepsilon^{2} \rho^{2}}\right) .
$$


Using our assumptions $\varepsilon=O\left(k^{2} \log n / \Lambda\right), \frac{\lambda^{2} \Lambda}{n}=O\left(\lambda^{2} \frac{k^{2} \log n}{n \varepsilon}\right)$ and therefore we conclude that

$$
\mathbb{E}\left[\hat{\delta}_{2}\left(\frac{1}{\hat{\rho}} \hat{B}, H_{n}(W)\right)^{2}\right] \leq O\left(\mathbb{E}\left[\hat{\varepsilon}_{k}^{(O)}\left(H_{n}(W)\right)^{2}\right]+\lambda\left(\frac{\log k}{\rho n}+\frac{k^{2}}{\rho n^{2}}\right)+\lambda^{2} \frac{k^{2} \log n}{n \varepsilon}+\frac{\lambda^{2}}{n^{2} \rho^{2} \varepsilon^{2}}\right) .
$$

Consider now $V$ a $k \times k$ matrix such that $\varepsilon_{k}^{(O)}(W)=\|W-W[V]\|_{2}$. Since $B$ is obtained by averages of the values of $W$ over $k$ classes we have that $\|V\|_{2} \leq\|W\|_{2} \leq \sqrt{\|W\|_{\infty}\|W\|_{1}} \leq \sqrt{\lambda}$. Furthermore by Lemma 6.5 for $\pi$ the standard $k$-equipartition of $[n]$ we have

$$
\varepsilon_{k}^{(O)}(W) \geq\left\|W-W\left[V_{\pi}\right]\right\|_{2}-\sqrt{\frac{10 \lambda(k-1)}{n}} \geq \hat{\delta}_{2}\left(V_{\pi}, W\right)-\sqrt{\frac{10 \lambda(k-1)}{n}} .
$$

Hence for the $V$ and $\pi$ chosen above,

$$
\begin{aligned}
\hat{\varepsilon}_{k}^{(O)}\left(H_{n}(W)\right) & \leq \hat{\delta}_{2}\left(V, H_{n}(W)\right) \\
& =\min _{\sigma \in \mathcal{S}_{n}}\left\|V_{\pi}-H_{n}(W)_{\sigma}\right\|_{2}, \text { using Lemma } 6 \text { in [15] } \\
& \leq\left\|W\left[V_{\pi}\right]-W\right\|_{2}+\hat{\delta}_{2}\left(H_{n}(W), W\right) \\
& \leq \varepsilon_{k}^{(O)}(W)+\varepsilon_{n}(W)+O\left(\sqrt{\frac{\lambda(k-1)}{n}}\right), \operatorname{using}[6.16
\end{aligned}
$$

Now using this bound and the elementary $(u+v+w)^{2} \leq 3\left(u^{2}+v^{2}+w^{2}\right)$ we obtain that $\mathbb{E}\left[\delta_{2}\left(\frac{1}{\hat{\rho}} \hat{B}, H_{n}(W)\right)^{2}\right]$ is at most

$$
O\left(\mathbb{E}\left[\varepsilon_{k}^{(O)}(W)^{2}+\varepsilon_{n}(W)^{2}\right]+\lambda \frac{k-1}{n}+\lambda\left(\frac{\log k}{\rho n}+\frac{k^{2}}{\rho n^{2}}\right)+\lambda^{2} \frac{k^{2} \log n}{n \varepsilon}+\frac{\lambda^{2}}{n^{2} \rho^{2} \varepsilon^{2}}\right) .
$$

Using now triangle inequality for $\delta_{2}$ we have

$$
\delta_{2}\left(\frac{1}{\hat{\rho}} \hat{B}, W\right) \leq \delta_{2}\left(\frac{1}{\hat{\rho}} \hat{B}, H_{n}(W)\right)+\delta_{2}\left(H_{n}(W), W\right)=\delta_{2}\left(\frac{1}{\rho} \hat{B}, H_{n}(W)\right)+\varepsilon_{n}(W)
$$

almost surely and therefore $\mathbb{E}\left[\delta_{2}\left(\frac{1}{\hat{\rho}} \hat{B}, W\right)^{2}\right]$ is at most of the order

$$
O\left(\mathbb{E}\left[\varepsilon_{k}^{(O)}(W)^{2}+\varepsilon_{n}(W)^{2}\right]+\lambda \frac{k-1}{n}+\lambda\left(\frac{\log k}{\rho n}+\frac{k^{2}}{\rho n^{2}}\right)+\lambda^{2} \frac{k^{2} \log n}{n \varepsilon}+\frac{\lambda^{2}}{n^{2} \rho^{2} \varepsilon^{2}}\right) .
$$

This completes the proof of Theorem 3.1 .

Proof of Corollary 3.2. Since $k \geq 2$ and $\rho n \geq k-2$ the lower bound follows from Theorem 3 from [44] for $r=\Lambda \rho$. For the upper bound we use Algorithm 1 from [15] and the output $k$ block graphon defined by $\frac{1}{\hat{\rho}} \hat{B}$. For any $k$-block graphon and $\lambda=O(\Lambda)$, Theorem 3.1 implies that $\mathbb{E}\left[\delta_{2}\left(\frac{1}{\hat{\rho}} \hat{B}, W\right)^{2}\right]$ is at most of the order

$$
O\left(\mathbb{E}\left[\varepsilon_{k}^{(O)}(W)^{2}+\varepsilon_{n}(W)^{2}\right]+\Lambda \frac{k-1}{n}+\Lambda\left(\frac{\log k}{\rho n}+\frac{k^{2}}{\rho n^{2}}\right)+\Lambda^{2} \frac{k^{2} \log n}{n \varepsilon}+\frac{\Lambda^{2}}{n^{2} \rho^{2} \varepsilon^{2}}\right) .
$$


Now, since $W$ is a $k$-block graphon we have $\varepsilon_{k}^{(O)}(W)=0$. Furthermore by Lemma 14 in [15], $\mathbb{E}\left[\varepsilon_{n}(W)^{2}\right] \leq O\left(\Lambda^{2} \sqrt{\frac{k}{n}}\right)$. Plugging both these equalities in (6.17) and using $\Lambda \frac{k-1}{n}=O\left(\Lambda^{2} \sqrt{\frac{k}{n}}\right)$ we conclude that for any $k$-block graphon $\mathbb{E}\left[\delta_{2}\left(\frac{1}{\hat{\rho}} \hat{B}, W\right)^{2}\right]$ is at most of the order

$$
O\left(\Lambda^{2} \sqrt{\frac{k}{n}}+\Lambda\left(\frac{\log k}{\rho n}+\frac{k^{2}}{\rho n^{2}}\right)+\Lambda^{2} \frac{k^{2} \log n}{n \varepsilon}+\frac{\Lambda^{2}}{n^{2} \rho^{2} \varepsilon^{2}}\right) .
$$

This completes the proof of the upper bound and the Corollary.

\section{Proof of Theorem 3.3}

Proof. For simplicity we present the proof only in the case $k=2$. The argument generalizes easily to higher $k$.

We claim that it suffices to be established that one cannot estimate the density of any $k$-block graphon $W,\|W\|_{1}$, with mean squared error of the order $O\left(\max \left(\frac{1}{n}, \frac{1}{\varepsilon^{2} n^{2}}\right)\right)$. We establish this claim by contradiction. Suppose that we have established the above and that the rate $\tilde{R}_{k}(\varepsilon, n)$ is $O\left(\frac{1}{n^{2} \varepsilon^{2}}\right)$. That implies the existence of an $\varepsilon$-node-DP algorithm, which for any $W k$-block graphon,

$$
\mathbb{E}_{G \sim G_{n}(\rho W), \hat{B} \sim \mathcal{A}_{G}}\left[\delta_{2}(\hat{B}, W)^{2}\right]=O\left(\frac{1}{n^{2} \varepsilon^{2}}\right) .
$$

Note though that by Cauchy-Schawrz and triangle inequality,

$$
\delta_{2}(\hat{B}, W)=\min _{\varphi}\left\|W[\hat{B}]_{\varphi}-W\right\|_{2} \geq \min _{\varphi}\left\|W[\hat{B}]_{\varphi}-W\right\|_{1} \geq\left|\left\|W[\hat{B}]_{\varphi}\right\|_{1}-\|W\|_{1}\right|,
$$

where $\varphi$ ranges over all measure-preserving transformations of $[0,1]$. In particular, for any $W$ $k$-block graphon,

$$
\mathbb{E}_{G \sim G_{n}(\rho W), \hat{B} \sim \mathcal{A}_{G}}\left[\left|\left\|W[\hat{B}]_{\varphi}\right\|_{1}-\|W\|_{1}\right|^{2}\right]=O\left(\left(\frac{1}{n^{2} \varepsilon^{2}}\right) .\right.
$$

This is a contradiction with the assumption that we cannot approximate the density of $W$ at a $O\left(\max \left(\frac{1}{n}, \frac{1}{\varepsilon^{2} n^{2}}\right)\right)$ level. The proof of the claim is complete.

Now we proceed with the lower bound on the density estimation by giving a reduction to (regular) differentially private estimation of the secret parameter $q$ given $n$ samples from a Bernouilli distribution (that is, $n$ biased coins, each of which is heads independently with probability $q$ ).

Given a parameter $q \in[0,1]$, let $W_{q}:[0,1]^{2} \rightarrow[0,1]$ be the graphon given by

$$
W_{q}(x, y)= \begin{cases}1 & \text { if } x, y \leq q \text { or } x, y \geq q \\ 0 & \text { otherwise }\end{cases}
$$

This is a 2-block graphon with blocks of sizes $q$ and $1-q$, respectively. The graphs generated from $W_{q}$ consist of two cliques (of size roughly $q n$ and $(1-q) n$ ) so it is easy to know which vertices belong to the same block. 
The density of $W_{q}$ is $\tau(q)=q^{2}+(1-q)^{2}=\frac{1}{2}+2\left(q-\frac{1}{2}\right)^{2}$. Consider an algorithm $A$ that, given $G \sim G_{n}(W)$, aims to estimate the density $\tau(q)$ of $W_{q}$. We can use its output (call it $\hat{\tau}$ ) to estimate

$q$ by setting $\hat{q}=\frac{1}{2}-\sqrt{\frac{\hat{\tau}-\frac{1}{2}}{2}}$. This function's derivative is finite and nonzero as long as $\hat{\tau}$ is bounded away from $\frac{1}{2}$. Thus, an algorithm that can estimate $\tau(q)$ within error $\alpha$ on samples from $W_{q}$ can be used to estimate $q$ up to error $O(\alpha)$ (as long as $q$ is bounded away from $1 / 2$ ).

To reduce to estimation of the Bernouilli parameter, suppose we are given a sample $X=$ $\left(X_{1}, X_{2}, \ldots, X_{n}\right)$ of size $n$, drawn i.i.d. from $\operatorname{Bernouilli}(q)$ for uknown $q$. We may generate a graph $G(X)$ by creating two cliques of size $N_{0}$ and $N_{1}$, respectively, where $N_{1}=\sum_{i} X_{i}$ (the number of ones in $X$ ) and $N_{0}=n-N_{1}$. The distribution of $G(X)$ is exactly $G_{n}\left(W_{q}\right)$, and so we can run our density estimation algorithm $A$ to get an estimate $\hat{\tau}$ of the density of $W_{q}$ and use that to compute $\hat{q}$, an estimate of $q$.

Observe that, if $A$ is $\varepsilon$-node-differentially private, then the composed algorithm $A(G(\cdot))$ is $\varepsilon$ differentially private with respect to its input (from $\{0,1\}^{n}$ ). To see why, note that changing one bit of the string $x$ changes the edges of exactly one vertex in $G(x)$ (corresponding to a change in the clique to which it is assigned). Since $A$ is $\varepsilon$-node-differentially private, a change in one bit of $x$ yields a change of at most $\varepsilon$ in the distribution of $A(G(x))$.

Fix a constant $c>0$ and consider the distribution $P$ obtained by choosing $q$ uniformly in $\frac{1}{4}, \frac{1}{4}+\alpha$, where $\alpha=c \max \left(\frac{1}{\sqrt{n}}, \frac{1}{\varepsilon n}\right)$ is the desired error bound, and outputing $W_{q}$. One can pick $c$ so that there is no $\varepsilon$-differentially private algorithm that can distinguish the corresponging Bernouilli distributions (with $q \in \frac{1}{4}, \frac{1}{4}+\alpha$ ) with probability better than 0.9 .

An $\varepsilon$-node-DP algorithm for estimating the density with error $o(\alpha)$ could be used to create an algorithm for Bernouilli estimation with error $o(\alpha)$, which would in turn allow one to estimate $q$ with error $o(\alpha)$, yielding a contradiction.

\section{Proof of Theorem 5.1}

We start with a lemma.

Lemma 8.1. Let $\mu$ be a probability measure on $\Omega$ and $\mathcal{A}^{\prime}$ be a randomized algorithm designed for input from $\mathcal{H}^{\prime} \subseteq \mathcal{M}$. Suppose that for any $D \in \mathcal{H}^{\prime}, \mathcal{A}^{\prime}(D)$ is absolutely continuous to $\mu$ and let $f_{D}$ the Radon-Nikodym derivative $\frac{d \mathcal{A}^{\prime}(D)}{d \mu}$. Then the following are equivalent

(1) $\mathcal{A}^{\prime}$ is $\varepsilon$-differentially private;

(2) For any $D, D^{\prime} \in \mathcal{H}$

$$
f_{\mathcal{A}^{\prime}(D)} \leq \exp \left(\varepsilon d\left(D, D^{\prime}\right)\right) f_{\mathcal{A}^{\prime}\left(D^{\prime}\right)},
$$

$\mu$-almost surely.

Proof. For the one direction, suppose $\mathcal{A}^{\prime}$ satisfies (8.1). Then for any set $S \in \mathcal{F}$ we obtain

$$
\begin{aligned}
\mathbb{P}\left(\mathcal{A}^{\prime}(D) \in S\right) & =\int_{S} f_{\mathcal{A}^{\prime}(D)} d \mu \\
& \leq \exp \left(\varepsilon d\left(D, D^{\prime}\right)\right) \int_{S} f_{\mathcal{A}^{\prime}\left(D^{\prime}\right)} d \mu \\
& =\exp \left(\varepsilon d\left(D, D^{\prime}\right)\right) \mathbb{P}\left(\mathcal{A}^{\prime}(D) \in S\right)
\end{aligned}
$$


We prove the other direction by contradiction. Consider the set

$$
S=\left\{f_{\mathcal{A}^{\prime}(D)}>\exp \left(\varepsilon d\left(D, D^{\prime}\right)\right) f_{\mathcal{A}^{\prime}\left(D^{\prime}\right)}\right\} \in \mathcal{F}
$$

and assume that $\mu(S)>0$. By definition on being strictly positive on a set of positive measure

$$
\int_{S}\left[f_{\mathcal{A}^{\prime}(D)}-\exp \left(\varepsilon d\left(D, D^{\prime}\right)\right) f_{\mathcal{A}^{\prime}\left(D^{\prime}\right)}\right] d \mu>0
$$

or equivalently

$$
\int_{S} f_{\mathcal{A}^{\prime}(D)} d \mu>\exp \left(\varepsilon d\left(D, D^{\prime}\right)\right) \int_{S} f_{\mathcal{A}^{\prime}\left(D^{\prime}\right)} d \mu
$$

On the other hand using $\varepsilon$-differential privacy we obtain

$$
\begin{aligned}
\int_{S} f_{\mathcal{A}^{\prime}(D)} d \mu & =\mathbb{P}\left(\mathcal{A}^{\prime}(D) \in S\right) \\
& \leq \exp \left(\varepsilon d\left(D, D^{\prime}\right)\right) \mathbb{P}\left(\mathcal{A}^{\prime}\left(D^{\prime}\right) \in S\right) \\
& =\exp \left(\varepsilon d\left(D, D^{\prime}\right)\right) \int_{S} f_{\mathcal{A}^{\prime}\left(D^{\prime}\right)} d \mu,
\end{aligned}
$$

a contradiction with (8.2). This completes the proof of the Lemma.

Now we establish Theorem 5.1 .

Proof. Since $\mathcal{H} \neq \varnothing$, let $D_{0} \in \mathcal{H}$ and denote by $\mu$ the measure $\hat{\mathcal{A}}\left(D_{0}\right)$. From the definition of differential privacy we know for all $D \in \mathcal{H}$ and $S \in \mathcal{F}$, if $\mathbb{P}\left(\hat{\mathcal{A}}\left(D_{0}\right) \in S\right)=0$ then $\mathbb{P}(\hat{\mathcal{A}}(D) \in S)=$ 0 . In the language of measure theory that means the measure $\hat{\mathcal{A}}(D)$ is absolutely continuous to $\mathcal{A}\left(D_{0}\right)$. By Radon-Nikodym theorem we conclude that there are measurable functions $f_{D}: \Omega \rightarrow$ $[0,+\infty)$ such that for all $S \in \mathcal{F}$,

$$
\mathbb{P}(\hat{\mathcal{A}}(D) \in S)=\int_{S} f_{D} d \mu
$$

We define now the following randomized algorithm $\mathcal{A}$. For every $D \in \mathcal{M}, \mathcal{A}(D)$ samples from $\Omega$ according to the absolutely continuous to $\mu$ distribution with density proportional to

$$
\inf _{D^{\prime} \in \mathcal{H}}\left[\exp \left(\varepsilon d\left(D, D^{\prime}\right)\right) f_{\hat{\mathcal{A}}\left(D^{\prime}\right)}\right] .
$$

That is for every $\omega \in \Omega$ its density with respect to $\mu$ is defined as

$$
f_{\mathcal{A}(D)}(\omega)=\frac{1}{Z_{D}} \inf _{D^{\prime} \in \mathcal{H}}\left[\exp \left(\varepsilon d\left(D, D^{\prime}\right)\right) f_{\hat{\mathcal{A}}\left(D^{\prime}\right)}(\omega)\right],
$$

where

$$
Z_{D}:=\int_{\Omega} \inf _{D^{\prime} \in \mathcal{H}}\left[\left(\varepsilon d\left(D, D^{\prime}\right)\right) f_{\hat{\mathcal{A}}(D)^{\prime}}\right] d \mu .
$$

In particular for all $S \in \mathcal{F}$ it holds

$$
\mathbb{P}(\mathcal{A}(D) \in S)=\int_{S} f_{\mathcal{A}(D)} d \mu .
$$


We first prove that $\mathcal{A}$ is $2 \varepsilon$-differentially private over all pairs of input from $\mathcal{M}$. Using Lemma 8.1 it suffices to prove that for any $D_{1}, D_{2} \in \mathcal{H}$,

$$
f_{\mathcal{A}\left(D_{1}\right)} \leq \exp \left(2 \varepsilon d\left(D_{1}, D_{2}\right)\right) f_{\mathcal{A}\left(D_{2}\right)}
$$

$\mu$-almost surely. We establish it in particular for every $\omega \in \Omega$. Let $D_{1}, D_{2} \in \mathcal{M}$. Using triangle inequality we obtain for every $\omega \in \Omega$,

$$
\begin{aligned}
\inf _{D^{\prime} \in \mathcal{H}}\left[\exp \left(\varepsilon d\left(D_{1}, D^{\prime}\right)\right) f_{\hat{\mathcal{A}}\left(D^{\prime}\right)}(\omega)\right] & \leq \inf _{D^{\prime} \in \mathcal{H}}\left[\exp \left(\varepsilon\left[d\left(D_{1}, D_{2}\right)+d\left(D_{2}, D^{\prime}\right)\right]\right) f_{\hat{\mathcal{A}}\left(D^{\prime}\right)}(\omega)\right] \\
& =\exp \left(\varepsilon d\left(D_{1}, D_{2}\right)\right) \inf _{D^{\prime} \in \mathcal{H}}\left[\exp \left(\varepsilon d\left(D, D^{\prime}\right)\right) f_{\hat{\mathcal{A}}\left(D^{\prime}\right)}(\omega)\right],
\end{aligned}
$$

which implies that for any $D_{1}, D_{2} \in \mathcal{M}$,

$$
\begin{aligned}
Z_{D_{1}} & =\int_{\Omega} \inf _{D^{\prime} \in \mathcal{H}}\left[\exp \left(\varepsilon d\left(D_{1}, D^{\prime}\right)\right) f_{\hat{\mathcal{A}}\left(D^{\prime}\right)}\right] d \mu \\
& \leq \exp \left(\varepsilon d\left(D_{1}, D_{2}\right)\right) \int_{\Omega} \inf _{D^{\prime} \in \mathcal{H}}\left[\exp \left(\varepsilon d\left(D_{2}, D^{\prime}\right)\right) f_{\hat{\mathcal{A}}\left(D^{\prime}\right)}(\omega)\right] d \mu \\
& =\exp \left(\varepsilon d\left(D_{1}, D_{2}\right)\right) Z_{D_{2}} .
\end{aligned}
$$

Therefore using the above two inequalities we obtain that for any $D_{1}, D_{2} \in \mathcal{H}$ and $\omega \in \Omega$,

$$
\begin{aligned}
f_{\mathcal{A}\left(D_{1}\right)}(\omega) & =\frac{1}{Z_{D_{1}}} \inf _{D^{\prime} \in \mathcal{H}}\left[\exp \left(\varepsilon d\left(D_{1}, D^{\prime}\right)\right) f_{\hat{\mathcal{A}}\left(D^{\prime}\right)}(\omega)\right] \\
& \leq \frac{1}{\exp \left(-\varepsilon d\left(D_{2}, D_{1}\right)\right) Z_{D_{2}}} \exp \left(\varepsilon d\left(D_{1}, D_{2}\right)\right) \inf _{D^{\prime} \in \mathcal{H}}\left[\exp \left(\varepsilon d\left(D_{2}, D^{\prime}\right)\right) f_{\hat{\mathcal{A}}\left(D^{\prime}\right)}(\omega)\right] \\
& =\exp \left(2 \varepsilon d\left(D_{1}, D_{2}\right)\right) \frac{1}{Z_{D_{2}}} \inf _{D^{\prime} \in \mathcal{H}}\left[\exp \left(\varepsilon d\left(D_{2}, D^{\prime}\right)\right) f_{\hat{\mathcal{A}}\left(D^{\prime}\right)}(\omega)\right] \\
& =\exp \left(2 \varepsilon d\left(D_{1}, D_{2}\right)\right) f_{\mathcal{A}\left(D_{2}\right)}(\omega),
\end{aligned}
$$

as we wanted.

Now we prove that for every $D \in \mathcal{H}, \mathcal{A}(D) \stackrel{d}{=} \hat{\mathcal{A}}(D)$. Consider an arbitrary $D \in \mathcal{H}$. From Lemma 8.1 we obtain that $\hat{\mathcal{A}}$ is $\varepsilon$-differentially private which implies that for any $D, D^{\prime} \in \mathcal{H}$

$$
f_{\hat{\mathcal{A}}(D)} \leq \exp \left(\varepsilon d\left(D, D^{\prime}\right)\right) f_{\hat{\mathcal{A}}\left(D^{\prime}\right)},
$$

$\mu$-almost surely. Observing that the above inequality holds as $\mu$-almost sure equality if $D^{\prime}=D$ we obtain that for any $D \in \mathcal{H}$ it holds

$$
f_{\hat{\mathcal{A}}(D)}(x)=\inf _{D^{\prime} \in \mathcal{H}}\left[\exp \left(\varepsilon d\left(D, D^{\prime}\right)\right) f_{\hat{\mathcal{A}}\left(D^{\prime}\right)}(x)\right],
$$

$\mu$-almost surely. Using that $f_{\hat{\mathcal{A}}(D)}$ is the Radon-Nikodym derivative $\frac{d \hat{\mathcal{A}}(D)}{d \mu}$ we conclude

$$
Z_{D}:=\int_{\Omega} f_{\hat{\mathcal{A}}(D)} d \mu=\mu(\Omega)=1 .
$$

Therefore

$$
f_{\hat{\mathcal{A}}(D)}=\frac{1}{Z_{D}} \inf _{D^{\prime} \in \mathcal{H}}\left[\exp \left(\varepsilon d\left(D, D^{\prime}\right)\right) f_{\hat{\mathcal{A}}\left(D^{\prime}\right)}\right]
$$


$\mu$-almost surely and hence

$$
f_{\hat{\mathcal{A}}(D)}=f_{\mathcal{A}(D)},
$$

$\mu$-almost surely. This suffices to conclude that $\hat{\mathcal{A}}(D) \stackrel{d}{=} \mathcal{A}(D)$ as needed.

The proof of Theorem 5.1 is complete.

\section{The Proof of the $n^{\frac{3}{2}}$-Upper Bound}

Definitions and Notation Recall that $\mathcal{G}_{n}$ is the set of all undirected graphs on the vertex set $[n]$. For any $\rho \in[0,1]$ let $\mathcal{G}_{n \rho}$ the set of all undirected graphs on $n$ vertices with edge density at most $\rho$,

$$
\mathcal{G}_{n, \rho}=\left\{G \in \mathcal{G}_{n} \mid e(G) \leq \rho\right\} .
$$

For any $\varnothing \neq S \subseteq[n], t, \rho \in[0,1]$ and $C>0$,

$$
A_{\rho, S, C}(t):=\left\{G \in \mathcal{G}_{n, \rho}|| E\left(S, S^{c}\right)+E(S)-t\left[k(n-k)+\left(\begin{array}{l}
k \\
2
\end{array}\right)\right]\left|\leq C \max \left\{\sqrt{\rho}, \sqrt{\frac{\log n}{n}}\right\} k \sqrt{n \log n}\right|\right\} .
$$

Consider the "homogeneity" set

$$
\mathcal{H}_{\rho, C}=\bigcap_{\varnothing \neq S \subseteq[n]} A_{\rho, C, S}(e(G))
$$

\section{Auxilary Lemmata}

Lemma 9.1. Let $p \in[0,1] \cap \mathbb{Q}$. Suppose $N$ grows to infinity constrained to $N p \in \mathbb{Z}$. Then

$$
\left(\begin{array}{c}
N \\
N p
\end{array}\right) \exp \left(-N H_{2}(p)\right)=\Omega\left(\frac{1}{\sqrt{N}}\right)
$$

where $H_{2}(p)=-p \log p-(1-p) \log (1-p)$.

Proof. We have

$$
\left(\begin{array}{c}
N \\
N p
\end{array}\right)=\frac{N !}{(N p) !(N(1-p)) !} .
$$

By Stirling approximation $n !=\Theta\left(\left(\frac{n}{e}\right)^{n} \sqrt{2 \pi n}\right)$. Therefore

$$
\begin{aligned}
\left(\begin{array}{c}
N \\
N p
\end{array}\right) & =\Theta\left(\frac{\left(\frac{N}{e}\right)^{N} \sqrt{2 \pi N}}{\left[\left(\frac{N p}{e}\right)^{N p} \sqrt{2 \pi N p}\left(\frac{N(1-p)}{e}\right)^{N(1-p)} \sqrt{2 \pi N(1-p)}\right]}\right) \\
& =\Theta\left(\frac{1}{2 \sqrt{N p(1-p)}} \exp (-N(p \log p+(1-p) \log (1-p)))\right) \\
& =\Omega\left(\frac{1}{\sqrt{N}}\right) \exp \left(N H_{2}(p)\right) .
\end{aligned}
$$

or

$$
\left(\begin{array}{c}
N \\
N p
\end{array}\right) \exp \left(-N H_{2}(p)\right)=\Omega\left(\frac{1}{\sqrt{N}}\right) .
$$

The proof of the Lemma is complete. 
Lemma 9.2. For any $a, b>0$ the function $f: \mathbb{R} \rightarrow \mathbb{R}$, with $f(x)=\min \{a|x|, b\}$, for all $x \in \mathbb{R}$, satisfies the triangle inequality, $f(x+y) \leq f(x)+f(y)$ for all $x, y \in \mathbb{R}$.

Proof. Let $x, y \in \mathbb{R}$. We distinguish two cases.

If $a|x| \geq b$, or $a|y| \geq b$ then

$$
f(x+y) \leq b \leq \min \{a|x|, b\}+\min \{a|y|, b\}=f(x)+f(y) .
$$

If $b \leq a|x|$ and $|a| y \leq b$ then

$$
f(x+y) \leq a|x+y| \leq a|x|+a|y|=\min \{a|x|, b\}+\min \{a|y|, b\}=f(x)+f(y) .
$$

This completes the proof of the Lemma.

\section{Main Lemmata}

Lemma 9.3. Let $\rho \in[0,1], m \in \mathbb{N}$ with $m \leq \rho\left(\begin{array}{c}n \\ 2\end{array}\right), p=\frac{m}{\left(\begin{array}{c}n \\ 2\end{array}\right)} \in[0, \rho]$ and $C>48$. Then it holds

$$
\mathbb{P}_{G \sim G_{n, p}}\left[\mathcal{H}_{\rho, C}^{c}\right]=O\left(\frac{1}{n^{\frac{C}{16}-2}}\right) .
$$

Furthermore

$$
\mathbb{P}_{G \sim G(n, m)}\left[\mathcal{H}_{\rho, C}^{c}\right]=O\left(\frac{1}{n^{\frac{C}{16}-3}}\right) .
$$

Proof. We start with proving that

$$
\mathbb{P}_{G \sim G_{n, p}}\left[\mathcal{H}_{\rho, C}^{c}\right] \leq \mathbb{P}_{G \sim G_{n, p}}\left(|e(G)-p|>\frac{C \sqrt{\rho \log n}}{2 \sqrt{n}}\right)+\mathbb{P}_{G \sim G_{n, p}}\left(\bigcup_{\varnothing \neq S \subseteq[n]} A_{\rho, S, \frac{C}{2}}^{c}(p)\right)
$$

To establish (9.1) it suffices to establish that

$$
\mathbb{P}_{G \sim G_{n, p}}\left(\mathcal{H}_{\rho, C}^{c} \cap\left\{|e(G)-p| \leq \frac{C \sqrt{\rho \log n}}{2 \sqrt{n}}\right\}\right) \leq \mathbb{P}_{G \sim G_{n, p}}\left(\bigcup_{\varnothing \neq S \subseteq[n]} A_{\rho, S, \frac{C}{2}}^{c}(p)\right) .
$$

We establish the corresponding set inclusion. Let $G \in \mathcal{H}_{\rho, C}^{c} \cap\left\{|e(G)-p| \leq C \frac{\sqrt{\rho \log n}}{2 \sqrt{n}}\right\}$. Since $G \in \mathcal{H}_{\rho, C}^{c}$ there exists $\varnothing \neq S \subseteq[n]$ with $G \in A_{\rho, S, C}^{c}(e(G))$ and therefore it holds

$$
E\left(S, S^{c}\right)+E(S)-e(G)\left[k(n-k)+\left(\begin{array}{l}
k \\
2
\end{array}\right)\right] \mid>C \max \left\{\sqrt{\rho}, \sqrt{\frac{\log n}{n}}\right\} k \sqrt{n \log n} .
$$

Since $|e(G)-p| \leq \frac{C \sqrt{\rho \log n}}{2 \sqrt{n}}$ we obtain

$$
\begin{aligned}
(p-e(G))\left[k(n-k)+\left(\begin{array}{l}
k \\
2
\end{array}\right)\right] & \leq \frac{C \sqrt{\rho \log n}}{2 \sqrt{n}}\left[k(n-k)+\left(\begin{array}{l}
k \\
2
\end{array}\right)\right] \\
& \leq \frac{C \sqrt{\rho \log n}}{2 \sqrt{n}} k n \\
& =\frac{C}{2} k \sqrt{\rho} \sqrt{n \log n} \\
& \leq \frac{C}{2} \max \left\{\sqrt{\rho}, \sqrt{\frac{\log n}{n}}\right\} k \sqrt{n \log n}
\end{aligned}
$$


Rearranging we have

$$
\frac{C}{2} \max \left\{\sqrt{\rho}, \sqrt{\frac{\log n}{n}}\right\} k \sqrt{n \log n}-p\left[k(n-k)+\left(\begin{array}{l}
k \\
2
\end{array}\right)\right] \geq-e(G)\left[k(n-k)+\left(\begin{array}{l}
k \\
2
\end{array}\right)\right] .
$$

Using this into (9.2) we obtain

$$
E\left(S, S^{c}\right)+E(S)-p\left[k(n-k)+\left(\begin{array}{l}
k \\
2
\end{array}\right)\right]>\frac{C}{2} \max \left\{\sqrt{\rho}, \sqrt{\frac{\log n}{n}}\right\} k \sqrt{n \log n},
$$

which means that indeed

$$
G \in A_{\rho, S, \frac{C}{2}}^{c}(p) \subseteq \bigcup_{\varnothing \neq S \subseteq[n]} A_{\rho, S, \frac{C}{2}}^{c}(p) .
$$

The proof of (9.1) is complete.

Now by Hoeffding inequality since $p \in[0, \rho]$, we have

$$
\mathbb{P}_{G \sim G_{n, p}}\left(|e(G)-p|>\frac{C \sqrt{\rho \log n}}{2 \sqrt{n}}\right) \leq 2 \exp \left(-\Omega\left(C^{2} n \log n\right)\right) .
$$

Combining (9.3) with (9.1) we conclude that to establish our result for the $G_{n, p}$ it suffices to establish

$$
\mathbb{P}_{G \sim G_{n, p}}\left(\bigcup_{\varnothing \neq S \subseteq[n]} A_{\rho, S, \frac{C}{2}}^{c}(p)\right)=O\left(\frac{1}{n^{\frac{C}{16}-2}}\right) .
$$

Now we recall that Bernstein inequality implies that for $N \in \mathbb{N}, Z$ distributed according to a $\operatorname{Bin}(N, p)$ and $t>0$,

$$
\mathbb{P}[|Z-N p| \geq t] \leq 2 \exp \left(-\frac{\frac{t^{2}}{2}}{N p+\frac{1}{3} t}\right) .
$$

Set $c_{0}=\max \left\{\sqrt{\rho}, \sqrt{\frac{\log n}{n}}\right\}$ and fix some $\varnothing \neq S \subseteq V(G)$. The random variable $E\left(S, S^{c}\right)+E(S)$ is distributed according to a $\operatorname{Bin}(N, p)$ for $N=k(n-k)+\left(\begin{array}{c}k \\ 2\end{array}\right)$. Therefore from (9.5) we have

$$
\mathbb{P}\left[A_{\rho, S, \frac{C}{2}}^{c}(p)\right] \leq 2 \exp \left(-\frac{C^{2}}{4} \frac{c_{0}^{2} k^{2} n \log n / 2}{\left(k(n-k)+\left(\begin{array}{c}
k \\
2
\end{array}\right)\right) p+C c_{0} k \sqrt{n \log n} / 6}\right) .
$$

Now observe that since $C>1$,

$$
\left(k(n-k)+\left(\begin{array}{l}
k \\
2
\end{array}\right)\right) p \leq k n p \leq C k n p \leq C k n \rho
$$

and by definition of $c_{0}$,

$$
c_{0} k \sqrt{n \log n} / 3 \leq \max \{k \sqrt{\rho n \log n}, k \log n\} .
$$

Furthermore notice that for any $\rho \in[0,1]$,

$$
k \sqrt{\rho n \log n} \leq \max \{\rho k n, k \log n\} .
$$


Therefore, using again that $C>1$,

$$
\left(k(n-k)+\left(\begin{array}{l}
k \\
2
\end{array}\right)\right) p+c_{0} k \sqrt{n \log n} / 3 \leq 2 C \max \{k n \rho, k \log n\} .
$$

Hence

$$
\begin{aligned}
\frac{c_{0}^{2} k n / 2}{\left(k(n-k)+\left(\begin{array}{l}
k \\
2
\end{array}\right)\right) p+C c_{0} k \sqrt{n \log n} / 6} & \geq \frac{c_{0}^{2} k n / 2}{2 C \max \{k n \rho, k \sqrt{\rho n \log n}, k \log n\}} \\
& \geq \frac{\max \{\rho k n, k \log n\} / 2}{2 C \max \{k n \rho, k \sqrt{\rho n \log n}, k \log n\}}, \text { from the definition of } c_{0}, \\
& \geq \frac{1}{4 C} .
\end{aligned}
$$

Using the last inequality in (9.6) we conclude that for any $\varnothing \neq S \subseteq V(G)$

$$
\mathbb{P}\left[A_{S, \rho, \frac{C}{2}}^{c}\right] \leq 2 \exp (-C k \log n / 16)=2 n^{-\frac{C k}{16}} .
$$

Using a union bound we obtain

$$
\begin{aligned}
& \mathbb{P}_{G \sim G_{n, p}}\left[\bigcup_{S \subseteq V(G)} A_{S, \rho, \frac{C}{2}}^{c}\right] \\
& \leq 2 \sum_{k=1}^{n}\left(\begin{array}{l}
n \\
k
\end{array}\right) n^{-\frac{C k}{16}} \\
& \leq 2 \sum_{k=1}^{n} n^{k} n^{-\frac{C k}{16}} \\
& \left.\leq 2 n \frac{1}{n^{\frac{C}{16}}-1}, \quad \text { using that } C>16\right) \\
& =\frac{2}{n^{\frac{C}{16}-2}} \\
& =O\left(\frac{1}{n^{\frac{C}{16}-2}}\right) .
\end{aligned}
$$

This completes the proof for the Erdos Renyi case.

For the $G(n, m)$ case, we first recall that for any $p_{1} \in[0,1]$ and $m_{1} \in \mathbb{Z}$ the distribution of an Erdos Renyi graph with parameter $p_{1}$, conditional on having exactly $m_{1}$ edges, is a sample from $G\left(n, m_{1}\right)$. Therefore using the tower property and the property already established for the Erdos Renyi case we conclude

$$
\underset{G \sim G_{n, p}}{\mathbb{E}}\left[\mathbb{P}_{G_{1} \sim G_{n, E(G)}}\left[\mathcal{H}_{\rho, C}^{c}\right]\right]=\mathbb{P}_{G \sim G_{n, p}}\left[\mathcal{H}_{\rho, C}^{c}\right] \leq 2 \frac{1}{n^{\frac{C}{16}-2}}
$$

Using Markov's inequality we obtain

$$
\mathbb{P}_{G_{1} \sim G(n, m)}\left[\mathcal{H}_{\rho, C}^{c}\right] \mathbb{P}_{G \sim G_{n, p}}[E(G)=m] \leq O\left(\frac{1}{n^{\frac{C}{16}-2}}\right)
$$


Since $p=\frac{m}{\left(\begin{array}{c}n \\ 2\end{array}\right)}$ and $E(G)$ is distributed according to a binomial $\operatorname{Bin}\left(\left(\begin{array}{l}n \\ 2\end{array}\right), p\right)$ we have for $N=\left(\begin{array}{l}n \\ 2\end{array}\right)$,

$$
\begin{aligned}
\mathbb{P}_{G \sim G_{n, p}}[E(G)=m] & =\mathbb{P}_{Z \sim \operatorname{Bin}(N, p)}[Z=N p] \\
& =\left(\begin{array}{c}
N \\
p N
\end{array}\right) p^{N p}(1-p)^{N(1-p)} \\
& =\left(\begin{array}{c}
N \\
p N
\end{array}\right) \exp \left(-N H_{2}(p)\right) \\
& =\Omega\left(\frac{1}{\sqrt{N}}\right)(\text { using Lemma 9.1) } \\
& =\Omega\left(\frac{1}{n}\right) .
\end{aligned}
$$

Therefore

$$
\mathbb{P}_{G_{1} \sim G(n, m)}\left[\mathcal{H}_{\rho, C}^{c}\right] \Omega\left(\frac{1}{n}\right) \leq \mathbb{P}_{G_{1} \sim G(n, m)}\left[\mathcal{H}_{\rho, C}^{c}\right] \mathbb{P}_{G \sim G_{n, p}}[E(G)=m] \leq O\left(\frac{1}{n^{\frac{C}{16}-2}}\right)
$$

or

$$
\mathbb{P}_{G_{1} \sim G(n, m)}\left[\mathcal{H}_{\rho, C}^{c}\right] \leq O\left(\frac{1}{n^{\frac{C}{16}-3}}\right) .
$$

The proof of the Proposition is complete.

Lemma 9.4. Let $C>1$. For any $G, G^{\prime} \in \mathcal{H}_{\rho, C}$,

$$
\frac{1}{8 C} \min \left\{\frac{n^{\frac{3}{2}}}{\max \left\{\sqrt{\rho}, \sqrt{\frac{\log n}{n}}\right\} \sqrt{\log n}}\left|e(G)-e\left(G^{\prime}\right)\right|, n\right\} \leq d_{V}\left(G, G^{\prime}\right) .
$$

Proof. Let $G, G^{\prime} \in \mathcal{H}_{\rho, C}=\bigcap_{\varnothing \neq S \subseteq[n]} A_{S, \rho, C}$. Since $\frac{1}{8 C}<\frac{1}{4}$ we may assume without loss of generality that $d_{V}\left(G, G^{\prime}\right)<\frac{n}{4}$. It suffices to establish thatfor every pair $G, G^{\prime} \in H_{\rho}$ with $\delta_{V}\left(G, G^{\prime}\right)<\frac{n}{4}$ it holds

$$
\frac{1}{4 C} \frac{n^{\frac{3}{2}}}{\sqrt{\log n}}\left|e(G)-e\left(G^{\prime}\right)\right| \leq \max \left\{\sqrt{\rho}, \sqrt{\frac{\log n}{n}}\right\} d_{V}\left(G, G^{\prime}\right)
$$

Let $k:=d_{V}\left(G, G^{\prime}\right)$ and assume without loss of generality $k \geq 1$. By definition of the nodedistance there exist a non-empty subset of the vertices $S_{0} \subseteq[n]$ with $\left|S_{0}\right|=k$ so that we can construct $G$ from $G^{\prime}$ if we rewire only vertices belonging to the set $S_{0}$. From this property we conclude that the induced subgraphs defined only for the vertices of $S_{0}^{c}$ in $G$ and in $G^{\prime}$ are isomorphic. In particular

$$
E_{G}\left(S_{0}^{c}, S_{0}^{c}\right)=E_{G^{\prime}}\left(S_{0}^{c}, S_{0}^{c}\right)
$$

and therefore

$$
\left|E(G)-E\left(G^{\prime}\right)\right|=\left|E_{G}\left(S_{0}, S_{0}\right)+E_{G}\left(S_{0}, S_{0}^{c}\right)-E_{G^{\prime}}\left(S_{0}, S_{0}\right)-E_{G^{\prime}}\left(S_{0}, S_{0}^{c}\right)\right| .
$$

Since $G, G^{\prime} \in A_{\rho, S_{0}, C}$ we have

$$
\left|E_{G}\left(S_{0}, S_{0}\right)+E_{G}\left(S_{0}, S_{0}^{c}\right)-e(G)\left(k(n-k)+\left(\begin{array}{l}
k \\
2
\end{array}\right)\right)\right| \leq C \max \left\{\sqrt{\rho}, \sqrt{\frac{\log n}{n}}\right\} k \sqrt{n \log n}
$$


and

$$
\left|E_{G^{\prime}}\left(S_{0}, S_{0}\right)+E_{G^{\prime}}\left(S_{0}, S_{0}^{c}\right)-e\left(G^{\prime}\right)\left(k(n-k)+\left(\begin{array}{l}
k \\
2
\end{array}\right)\right)\right| \leq C \max \left\{\sqrt{\rho}, \sqrt{\frac{\log n}{n}}\right\} k \sqrt{n \log n} .
$$

Therefore by triangle inequality

$$
\left|E(G)-E\left(G^{\prime}\right)\right| \leq\left|e(G)-e\left(G^{\prime}\right)\right|\left(k(n-k)+\left(\begin{array}{l}
k \\
2
\end{array}\right)\right)+2 C \max \left\{\sqrt{\rho}, \sqrt{\frac{\log n}{n}}\right\} k \sqrt{n \log n},
$$

which from the definition of edge density can be rewritten as

$$
\left(\left(\begin{array}{l}
n \\
2
\end{array}\right)-\left(k(n-k)+\left(\begin{array}{l}
k \\
2
\end{array}\right)\right)\right)\left|e(G)-e\left(G^{\prime}\right)\right| \leq 2 C \max \left\{\sqrt{\rho}, \sqrt{\frac{\log n}{n}}\right\} k \sqrt{n \log n}
$$

Since $k(n-k)+\left(\begin{array}{l}k \\ 2\end{array}\right) \leq k n$ we have

$$
\left(\left(\begin{array}{l}
n \\
2
\end{array}\right)-k n\right)\left|e(G)-e\left(G^{\prime}\right)\right| \leq C \max \left\{\sqrt{\rho}, \sqrt{\frac{\log n}{n}}\right\} k \sqrt{n \log n}
$$

But now as we have assumed $k \leq \frac{n}{4}-1$ we have $\left(\begin{array}{l}n \\ 2\end{array}\right)-k n \geq \frac{n(n-1)}{2}-n\left(\frac{n}{4}-1\right) \geq \frac{n^{2}}{4}$ and therefore

$$
\frac{n^{2}}{4}\left|e(G)-e\left(G^{\prime}\right)\right| \leq 2 C \max \left\{\sqrt{\rho}, \sqrt{\frac{\log n}{n}}\right\} k \sqrt{n \log n} .
$$

or

$$
\frac{1}{8 C} \frac{n^{\frac{3}{2}}}{\sqrt{\log n}}\left|e(G)-e\left(G^{\prime}\right)\right| \leq \max \left\{\sqrt{\rho}, \sqrt{\frac{\log n}{n}}\right\} k=\max \left\{\sqrt{\rho}, \sqrt{\frac{\log n}{n}}\right\} \delta_{V}\left(G, G^{\prime}\right),
$$

where in the last inequality we have used the definition of $k$. The proof of the Lemma is complete.

Construction of the Algorithm and First Results We now present the Algorithm that implies both the bounds of the Proposition 4.2 and the Theorem 4.3 .

Let $C>48$. We first define the algorithm $\hat{\mathcal{A}}$ only for input graphs $G$ belonging in $\mathcal{H}_{\rho, C}$. For $G \in \mathcal{H}_{\rho, C}, \hat{\mathcal{A}}(G)$ samples from a continuous distribution on $[0,1]$ which adds truncated Laplacian noise to the edge density. Specifically for $q \in[0,1]$ the density of the output distribution is given by

$$
f_{\mathcal{A}(G)}(q)=\frac{1}{Z_{\mathcal{A}(G)}} \exp \left(-\frac{\varepsilon}{2} \frac{1}{8 C} \min \left\{\frac{n^{\frac{3}{2}}}{\max \left\{\sqrt{\rho}, \sqrt{\frac{\log n}{n}}\right\} \sqrt{\log n}}|e(G)-q|, n\right\}\right),
$$

where

$$
Z_{\hat{\mathcal{A}}(G)}=\int_{0}^{1} \exp \left(-\frac{\varepsilon}{2} \frac{1}{8 C} \min \left\{\frac{n^{\frac{3}{2}}}{\max \left\{\sqrt{\rho}, \sqrt{\frac{\log n}{n}}\right\} \sqrt{\log n}}|e(G)-q|, n\right\}\right) d q .
$$

Lemma 9.5. The algorithm $\hat{\mathcal{A}}$ defined for graphs from $\mathcal{H}_{\rho, C}$ is $\varepsilon / 2$-node-DP. Furthermore, for any $G \in \mathcal{H}_{\rho, C}$,

$$
\mathbb{E}\left[(\hat{\mathcal{A}}(G)-e(G))^{2}\right]=O\left(\max \left\{\rho, \frac{\log n}{n}\right\} \frac{\log n}{n^{3} \varepsilon^{2}}\right) .
$$


Proof. Let $G, G^{\prime} \in \mathcal{H}_{\rho, C}$. Since both the distribution of $\hat{\mathcal{A}}(G)$ and $\hat{\mathcal{A}}\left(G^{\prime}\right)$ are continuous on $[0,1]$ and therefore absolutely continuous with respect to the Lebesqure measure on $[0,1]$ it suffices to check based on Lemma 8.1 that

$$
f_{\hat{\mathcal{A}}(G)}(q) \leq \exp \left(\frac{\varepsilon}{2} d_{V}\left(G, G^{\prime}\right)\right) f_{\hat{\mathcal{A}}\left(G^{\prime}\right)}(q),
$$

for all $q \in[0,1]$. Fix some $q \in[0,1]$. Then (9.13) after taking logarithms and reaaranging is equivalent with

$$
\min \left\{\frac{n^{\frac{3}{2}}}{\max \left\{\sqrt{\rho}, \sqrt{\frac{\log n}{n}}\right\} \sqrt{\log n}}\left|e\left(G^{\prime}\right)-q\right|, n\right\}-\min \left\{\frac{n^{\frac{3}{2}}}{\max \left\{\sqrt{\rho}, \sqrt{\frac{\log n}{n}}\right\} \sqrt{\log n}}|e(G)-q|, n\right\}
$$

being upper bounded by $8 C d_{V}\left(G, G^{\prime}\right)$. But Lemma 9.2 implies that (9.14) is upper bounded by

$$
\min \left\{\frac{n^{\frac{3}{2}}}{\max \left\{\sqrt{\rho}, \sqrt{\frac{\log n}{n}}\right\} \sqrt{\log n}}\left|e(G)-e\left(G^{\prime}\right)\right|, n\right\}
$$

Since $G, G^{\prime} \in \mathcal{H}_{\rho, C}$ by Lemma 9.4 the last quantity is upper bounded by $8 C d_{V}\left(G, G^{\prime}\right)$, as we wanted.

For the second part, we first ease the notation by setting $r_{n}=\frac{n^{\frac{3}{2}}}{\max \left\{\sqrt{\rho}, \sqrt{\frac{\log n}{n}}\right\} \sqrt{\log n}}$. Now we first bound the normalizing quantity $Z_{\hat{\mathcal{A}}(G)}$.

$$
\begin{aligned}
Z_{\hat{\mathcal{A}}(G)} & =\int_{0}^{1} \exp \left(-\frac{\varepsilon}{8 C} \min \left\{r_{n}|e(G)-q|, n\right\}\right) d q \\
& \geq \int_{0}^{1} \exp \left(-\frac{\varepsilon}{16 C} r_{n}|e(G)-q|\right) d q \\
& \geq \frac{16 C}{r_{n} \varepsilon} \int_{e(G)-1}^{e(G)} \exp (-|u|) d u\left(\text { for } u=\frac{\varepsilon}{8 C} r_{n}(e(G)-q)\right) \\
& \geq \frac{16 C}{r_{n} \varepsilon} \int_{-1}^{1} \exp (-1) d u \\
& =\Omega\left(\frac{1}{r_{n} \varepsilon}\right) .
\end{aligned}
$$

Therefore

$$
Z_{\hat{\mathcal{A}}(G)}=\Omega\left(\frac{1}{r_{n} \varepsilon}\right) .
$$

Next we bound the expected value as following: 


$$
\begin{aligned}
\mathbb{E}\left[(\hat{\mathcal{A}}(G)-e(G))^{2}\right] & =\int_{0}^{1}(q-e(G))^{2} f_{\hat{\mathcal{A}}(G)}(q) d q \\
& \left.=\frac{1}{Z_{\hat{\mathcal{A}}(G)}} \int_{0}^{1}(q-e(G))^{2} \exp \left(-\frac{\varepsilon}{8 C} r_{n}|e(G)-q|, n\right\}\right) d q \\
& \leq \frac{1}{Z_{\hat{\mathcal{A}}(G)}} \int_{-1}^{1} u^{2} \exp \left(-\frac{\varepsilon}{8 C} \min \left\{r_{n}|u|, n\right\}\right) d u,(\text { for } u=q-e(G)) \\
& \leq \frac{1}{Z_{\hat{\mathcal{A}}(G)}}\left(2 \exp \left(-\frac{n \varepsilon}{8 C}\right)+\int_{|u|<\min \left\{n / r_{n}, 1\right\}} u^{2} \exp \left(-\frac{\varepsilon}{8 C} r_{n}|u|\right) d u\right) \\
& \leq \frac{1}{Z_{\hat{\mathcal{A}}(G)}}\left(2 \exp \left(-\frac{n \varepsilon}{8 C}\right)+\int_{|u|<1} u^{2} \exp \left(-\frac{\varepsilon}{8 C} r_{n}|u|\right) d u\right) \\
& =\frac{1}{Z_{\hat{\mathcal{A}}(G)}}\left(2 \exp \left(-\frac{n \varepsilon}{8 C}\right)+O\left(\int_{|x|<r_{n}} \frac{x^{2}}{\varepsilon^{3} r_{n}^{3}} \exp (-|x|) d u\right)\right)\left(\text { for } x=\varepsilon r_{n} u\right) \\
& =\frac{1}{Z_{\hat{\mathcal{A}}(G)}} O\left(\exp \left(-\frac{n \varepsilon}{8 C}\right)+\frac{1}{\varepsilon^{3} r_{n}^{3}}\right)
\end{aligned}
$$

Using now the bound (9.15),

$$
\mathbb{E}\left[(\hat{\mathcal{A}}(G)-e(G))^{2}\right]=O\left(r_{n} \varepsilon \exp \left(-\frac{n \varepsilon}{8 C}\right)+\frac{1}{\varepsilon^{2} r_{n}^{2}}\right)
$$

Now we claim that because $\varepsilon n \rightarrow+\infty$ we have

$$
r_{n} \varepsilon \exp \left(-\frac{n \varepsilon}{8 C}\right)=o\left(\frac{1}{\varepsilon^{2} r_{n}^{2}}\right) .
$$

Indeed it suffices to show that

$$
r_{n}^{3} \varepsilon^{3} \exp \left(-\frac{n \varepsilon}{8 C}\right)=o(1) .
$$

Taking logarithms it suffices to show

$$
\log r_{n}+\log \varepsilon \ll n \varepsilon
$$

Since $r_{n}=O\left(n^{3}\right)$, it suffices

$$
\log (n) \ll n \varepsilon,
$$

which is true as we assume $\varepsilon n / \log n \rightarrow+\infty$. Therefore

$$
\mathbb{E}\left[(\hat{\mathcal{A}}(G)-e(G))^{2}\right]=O\left(\frac{1}{\varepsilon^{2} r_{n}^{2}}\right) .
$$

Plugging in the value of $r_{n}$ we conclude

$$
\mathbb{E}\left[(\hat{\mathcal{A}}(G)-e(G))^{2}\right]=O\left(\max \left\{\rho, \frac{\log n}{n}\right\} \frac{\log n}{n^{3} \varepsilon^{2}}\right) .
$$

The proof of the Lemma is complete. 
Since by Lemma 9.5, the algorithm $\hat{\mathcal{A}}$ is $\frac{\varepsilon}{2}$-node-DP on graphs from $\mathcal{H}_{\rho, C}$, using Theorem 5.1 we can extend it to an algorithm $\mathcal{A}$ which is defined on every graph on $n$ vertices such that

- $\mathcal{A}$ is $\varepsilon$-node-DP;

- For every $G \in \mathcal{H}_{\rho, C}, \mathcal{A}(G) \stackrel{d}{=} \hat{\mathcal{A}}(G)$.

This is the algorithm that we analyze to establish both Proposition 4.2 and Theorem 4.3 .

\section{Proof of Proposition 4.2 and Theorem 4.3}

Proof of Proposition 4.2. Fix $C>0$ large enough constant ( $C>48$ suffices for our initial choice). We claim that $\mathcal{A}$ satisfies the necessary property. Fix $m<\rho\left(\begin{array}{l}n \\ 2\end{array}\right)$. We first split the expected squared error depending on whether the samples graph depends on $\mathcal{H}_{\rho, C}$ or not.

$$
\begin{aligned}
& \underset{G \sim G(n, m)}{\mathbb{E}}\left[(\hat{\mathcal{A}}(G)-e(G))^{2}\right] \\
& =\underset{G \sim G(n, m)}{\mathbb{E}}\left[(\hat{\mathcal{A}}(G)-e(G))^{2} 1\left(G \notin H_{\rho, C}\right)\right]+\underset{G \sim G(n, m)}{\mathbb{E}}\left[(\hat{\mathcal{A}}(G)-e(G))^{2} 1\left(G \in H_{\rho, C}\right)\right]
\end{aligned}
$$

For the first term we have from Lemma 9.3 that

$$
\underset{G \sim G(n, m)}{\mathbb{E}}\left[(\hat{\mathcal{A}}(G)-e(G))^{2} 1\left(G \notin \mathcal{H}_{\rho, C}\right)\right] \leq \mathbb{P}_{G \sim G(n, m)}\left[G \notin \mathcal{H}_{\rho, C}\right]=O\left(n^{-\left(\frac{C}{16}-3\right)}\right) .
$$

For the second term we have from Lemma 9.4 that

$$
\begin{aligned}
\underset{G \sim G(n, m)}{\mathbb{E}}\left[(\hat{\mathcal{A}}(G)-e(G))^{2} 1\left(G \in \mathcal{H}_{\rho, C}\right)\right] & \leq \max _{G \in H} \underset{G \sim G(n, m)}{\mathbb{E}}\left[(\hat{\mathcal{A}}(G)-e(G))^{2}\right] \\
& \leq O\left(\max \left\{\rho, \frac{\log n}{n}\right\} \frac{\log n}{n^{3} \varepsilon^{2}}\right) .
\end{aligned}
$$

Combining the above we conclude that for any $C>48$,

$$
\underset{G \sim G(n, m)}{\mathbb{E}}\left[(\hat{\mathcal{A}}(G)-e(G))^{2}\right]=O\left(n^{-\left(\frac{C}{16}-3\right)}+\max \left\{\rho, \frac{\log n}{n}\right\} \frac{\log n}{n^{3} \varepsilon^{2}}\right) .
$$

Since $\varepsilon<1$, by choosing $C$ sufficiently large but constant we conclude

$$
\underset{G \sim G(n, m)}{\mathbb{E}}\left[(\hat{\mathcal{A}}(G)-e(G))^{2}\right]=O\left(\max \left\{\rho, \frac{\log n}{n}\right\} \frac{\log n}{n^{3} \varepsilon^{2}}\right) .
$$

This completes the proof.

Proof of Theorem 4.3. Fix a $C>0$ large enough constant $(C>48$ suffices as an initial choice). To prove the upper bound on the rate we discuss the performance of the algorithm $\mathcal{A}$ defined above. To bound its mean squared error consider a $p \in[0, \rho]$. We first use the bias-variance decomposition to get,

$$
\underset{G \sim G_{n, p}}{\mathbb{E}}\left[(\hat{\mathcal{A}}(G)-p)^{2}\right]=\underset{G \sim G_{n, p}}{\mathbb{E}}\left[(\hat{\mathcal{A}}(G)-e(G))^{2}\right]+\underset{G \sim G_{n, p}}{\mathbb{E}}\left[(e(G)-p)^{2}\right]
$$


The second term is the variance of the edge density and therefore

$$
\underset{G \sim G_{n, p}}{\mathbb{E}}\left[(e(G)-p)^{2}\right]=O\left(\frac{p}{n^{2}}\right)=O\left(\frac{\rho}{n^{2}}\right) .
$$

For the first term, we recall that a sample from $G_{n, p}$ conditional on having a fixed number of edges $m$, is distributed according to $G(n, m)$. Therefore

$$
\underset{G \sim G_{n, p}}{\mathbb{E}}\left[(\mathcal{A}(G)-e(G))^{2}\right]=\underset{G \sim G_{n, p}}{\mathbb{E}}\left[\underset{G^{\prime} \sim G(n, E(G))}{\mathbb{E}}\left[\left(\mathcal{A}\left(G^{\prime}\right)-e\left(G^{\prime}\right)\right)^{2}\right]\right] .
$$

Using Proposition 4.2 we conclude

$$
\underset{G \sim G_{n, p}}{\mathbb{E}}\left[(\mathcal{A}(G)-e(G))^{2}\right]=O\left(n^{-(C / 16-3)}+\max \left\{\rho, \frac{\log n}{n}\right\} \frac{\log n}{n^{3} \varepsilon^{2}}\right) .
$$

Combining the above we conclude that for any $C>48$,

$$
\underset{G \sim G_{n, p}}{\mathbb{E}}\left[(\hat{\mathcal{A}}(G)-p)^{2}\right]=O\left(\frac{\rho}{n^{2}}+\max \left\{\rho, \frac{\log n}{n}\right\} \frac{\log n}{n^{3} \varepsilon^{2}}+n^{-(C / 16-3)}\right) .
$$

Since $\varepsilon<1$ by choosing $C>0$ sufficiently large but constant we conclude

$$
\underset{G \sim G_{n, p}}{\mathbb{E}}\left[(\hat{\mathcal{A}}(G)-p)^{2}\right]=O\left(\frac{\rho}{n^{2}}+\max \left\{\rho, \frac{\log n}{n}\right\} \frac{\log n}{n^{3} \varepsilon^{2}}\right) .
$$

The proof of Theorem 4.3 is complete.

\section{Proof of Lemma 10}

Using Lemma 10 from [15] we have that the estimator $f(G)=e(G)+Z$, for $Z$ following $\operatorname{Lap}\left(\frac{4}{n \varepsilon}\right)$, is $\varepsilon$-node-DP. Therefore

$$
R_{1}(\rho, \varepsilon, n) \leq \mathbb{E}_{G \sim G_{n, p}}\left[(f(G)-p)^{2}\right]=O\left(\mathbb{E}_{G \sim G_{n, p}}\left[(e(G)-p)^{2}\right]+\mathbb{E}_{G \sim G_{n, p}}\left[Z^{2}\right] .\right.
$$

The first term is the variance of the edge density and therefore

$$
\mathbb{E}_{G \sim G_{n, p}}\left[(e(G)-p)^{2}\right]=O\left(\frac{\rho}{n^{2}}\right) .
$$

For the second term, we clearly have that it is of the order $O\left(\frac{1}{n^{2} \varepsilon^{2}}\right)$. Therefore we conclude

$$
\mathbb{E}_{G \sim G_{n, p}}\left[(f(G)-p)^{2}\right]=O\left(\frac{\rho}{n^{2}}+\frac{1}{n^{2} \varepsilon^{2}} .\right)
$$

The proof is complete. 


\section{Proofs for Proposition 4.4 and Theorem 4.5}

\section{Auxilary Lemmata}

Lemma 11.1. For $a, b, k \in \mathbb{N}$ with $a, b \rightarrow+\infty$ and $k=o(\min \{a, b\})$,

$$
\left(\begin{array}{l}
a \\
k
\end{array}\right) /\left(\begin{array}{l}
b \\
k
\end{array}\right)=\Theta\left(\left(\frac{a}{b}\right)^{k}\right)
$$

Proof. By Stirling approximation we have $n !=\Theta\left(\left(\frac{n}{e}\right)^{n} \sqrt{n}\right)$. Therefore as

$$
\begin{aligned}
\left(\begin{array}{l}
a \\
k
\end{array}\right) /\left(\begin{array}{l}
b \\
k
\end{array}\right) & =(a !(b-k) !) /(b !(a-k) !) \\
& =\Theta\left(\frac{a^{a}(b-k)^{b-k}}{b^{b}(a-k)^{a-k}} \sqrt{\frac{(a-k) b}{a(b-k)}}\right) \\
& =\Theta\left(\left(\frac{a}{b}\right)^{k}\left(1+\frac{k}{a-k}\right)^{a-k-\frac{1}{2}}\left(1+\frac{k}{b-k}\right)^{-(b-k)-\frac{1}{2}}\right) .
\end{aligned}
$$

Since $k=o(\min \{a, b\}),\left(1+\frac{k}{a-k}\right)^{a-k-\frac{1}{2}}=e+o(1)$ and $\left(1+\frac{k}{b-k}\right)^{-(b-k)-\frac{1}{2}}=e^{-1}+o(1)$. Combining them we conclude

$$
\left(1+\frac{k}{a-k}\right)^{a-k-\frac{1}{2}}\left(1+\frac{k}{b-k}\right)^{-(b-k)-\frac{1}{2}}=1+o(1)
$$

or

$$
\left.\Theta\left(\left(\frac{a}{b}\right)^{k}\left(1+\frac{k}{a-k}\right)^{a-k-\frac{1}{2}}\left(1+\frac{k}{b-k}\right)^{-(b-k)-\frac{1}{2}}\right)=\Theta\left(\frac{a}{b}\right)^{k}\right) .
$$

The proof is complete.

Lemma 11.2. Let $N=\left(\begin{array}{l}n \\ 2\end{array}\right)$ and $N \geq m \geq N / 3$. Let $G_{0}$ sampled from $P=G(n, m)$.

$$
\mathbb{P}_{G_{0} \sim P}\left(d_{\text {min }}^{G_{0}} \geq n / 5\right)=1-2^{-\Omega(n)} .
$$

Proof. It suffices to establish that for $v \in[n]$ arbitrary vertex in the graph,

$$
\mathbb{P}_{G_{0} \sim P}\left(d^{G_{0}}(v) \leq n / 5\right)=2^{-\Omega(n)} .
$$

Then the proof follows by a union bound over the set of vertices. By Pittel's inequality (see Section 1.4 in [52]) we can upper bound the probability by the corresponding probability for the Erdos-Renyi model multiplied by $O(\sqrt{m})$,

$$
\mathbb{P}_{G_{0} \sim P}\left(d^{G_{0}}(v) \leq n / 5\right) \leq 3 \sqrt{m} \mathbb{P}_{G_{0} \sim G_{n, m / N}}\left(d^{G_{0}}(v) \leq n / 5\right) .
$$

Since $m<n^{2}$, it suffices to establish

$$
\mathbb{P}_{G_{0} \sim G_{n, m / N}}\left(d^{G_{0}}(v) \leq n / 5\right)=2^{-\Omega(n)} .
$$

The distribution, though, of $d^{G_{0}}(v)$ in the Erdos Renyi model is a binomial with parameters $n-$ $1, p=m / N>1 / 3$. In particular, Hoeffding's inequality implies

$$
\mathbb{P}_{G_{0} \sim G_{n, m / N}}\left(d^{G_{0}}(v) \leq n / 5\right) \leq \mathbb{P}_{G_{0} \sim G_{n, m / N}}\left(\left|d^{G_{0}}(v)-\frac{m}{N}(n-1)\right| \geq \frac{2}{15} n\right)=2^{-\Omega(n)} .
$$

The proof is complete. 
Proof of Proposition 4.4. We consider two models. The first is $\mathbb{P}_{1}=P=G(n, m)$, that is a sample of a uniform graph on $n$ vertices and $m$ edges. The second is $\mathbb{P}_{2}=G(n, m, k)$ : sample first uniformly a graph according to $Q$, that is a uniform graph on $n$ vertices and $m+k$ edges. Then choose a uniformly chosen vertex $v$ of the graph and delete $\min \left\{d_{v}^{G_{0}}, k\right\}$ edges which are adjacent to the vertex, uniformly at random. Note that by $d_{v}^{G_{0}}$ we refer to the degree of the vertex in $G_{0}$. We claim that under the assumptions of our Proposition,

$$
\lim _{n \rightarrow+\infty} \operatorname{TV}\left(\mathbb{P}_{1}, \mathbb{P}_{2}\right)=0
$$

Note that after proving this we are done for the following reason. First, it implies that with probability tending to one there is a coupling between $\mathbb{P}_{1}$ and $\mathbb{P}_{2}$ such that they output the same graph with probability tending to one. Since $\mathbb{P}_{1}=P$ and $\mathbb{P}_{2}$ samples a graph from $Q$ and rewires a single vertex of the output graph, we conclude that there exists a coupling $(G, H)$ coming from $P$ and $Q$ respectively such that, with probability tending to one, one can obtain $G$ from $H$ by rewiring one vertex.

For the proof of (11.1) by Pinsker's inequality we have that

$$
\operatorname{TV}\left(\mathbb{P}_{1}, \mathbb{P}_{2}\right) \leq \sqrt{\mathrm{KL}\left(\mathbb{P}_{1}, \mathbb{P}_{2}\right)}
$$

Therefore, it suffices to prove that the KL divergence converges to zero or,

$$
\limsup _{n \rightarrow+\infty} \mathbb{E}_{G_{0} \sim \mathbb{P}_{1}}\left[\log \frac{\mathbb{P}_{1}\left[G=G_{0}\right]}{\mathbb{P}_{2}\left[G=G_{0}\right]}\right]=0 .
$$

For convenience, we focus on the equivalent

$$
\liminf _{n \rightarrow+\infty} \mathbb{E}_{G_{0} \sim \mathbb{P}_{1}}\left[\log \frac{\mathbb{P}_{2}\left[G=G_{0}\right]}{\mathbb{P}_{1}\left[G=G_{0}\right]}\right]=0 .
$$

By Jensen's inequality since log is concave, we have for all $n$,

$$
\mathbb{E}_{G_{0} \sim \mathbb{P}_{1}}\left[\log \frac{\mathbb{P}_{2}\left[G=G_{0}\right]}{\mathbb{P}_{1}\left[G=G_{0}\right]}\right] \leq \log \mathbb{E}_{G_{0} \sim \mathbb{P}_{1}}\left[\frac{\mathbb{P}_{2}\left[G=G_{0}\right]}{\mathbb{P}_{1}\left[G=G_{0}\right]}\right]=\log 1=0 .
$$

Therefore it suffices to show

$$
\liminf _{n \rightarrow+\infty} \mathbb{E}_{G_{0} \sim \mathbb{P}_{1}}\left[\log \frac{\mathbb{P}_{2}\left[G=G_{0}\right]}{\mathbb{P}_{1}\left[G=G_{0}\right]}\right] \geq 0 .
$$

Now for any $G_{0}$ on $n$ vertices with $m$ edges we lower bound $\mathbb{P}_{2}\left[G=G_{0}\right]$ as follows, 


$$
\begin{aligned}
\mathbb{P}_{2}\left[G=G_{0}\right] & =\sum_{G^{\prime} \text { with } \mathrm{m}+\mathrm{k} \text { edges }} \mathbb{P}\left(G^{\prime} \text { is chosen in the first step }\right) \mathbb{P}\left(G_{0} \mid G^{\prime}\right) \\
& =\sum_{G^{\prime} \text { with }} \frac{1}{\mathrm{~m}+\mathrm{k} \text { edges and } \mathbb{P}\left(G_{0} \mid G^{\prime}\right)>0} \mathbb{P}\left(G_{0} \mid G^{\prime}\right),\left(G^{\prime} \text { is chosen according to } Q .\right) \\
& =\frac{1}{\left(\begin{array}{c}
N \\
m+k
\end{array}\right)} \sum_{v \in V\left(G_{0}\right)} \mathbb{P}\left(G_{0} \mid G^{\prime}\right), \quad \text { is plausible by } G_{0} \text { via rewiring } v \\
& =\frac{1}{\left(\begin{array}{c}
N \\
m+k
\end{array}\right)} \sum_{v \in V\left(G_{0}\right)} \frac{1}{G^{\prime} \text { is plausible by } G_{0} \text { via rewiring } v\left(\begin{array}{l}
d^{G_{0}}(v)+k \\
k
\end{array}\right)} \\
& =\frac{1}{n\left(\begin{array}{c}
N \\
m+k
\end{array}\right)} \sum_{v \in V\left(G_{0}\right)} \frac{\left(\begin{array}{c}
n-d^{G_{0}}(v)-1 \\
k
\end{array}\right)}{\left(\begin{array}{c}
d^{G_{0}}(v)+k \\
k
\end{array}\right)}
\end{aligned}
$$

Since $\mathbb{P}_{1}\left[G=G_{0}\right]=\frac{1}{\left(\begin{array}{c}N \\ m\end{array}\right)}$, the ratio is equal to

$$
\frac{\mathbb{P}_{2}\left[G=G_{0}\right]}{\mathbb{P}_{1}\left[G=G_{0}\right]}=\frac{1}{n} \sum_{v \in V\left(G_{0}\right)} \frac{\left(\begin{array}{c}
n-d^{G_{0}}(v)-1 \\
k
\end{array}\right)}{\left(\begin{array}{c}
d^{G}(v)+k \\
k
\end{array}\right)}
$$

Set $\mathcal{E}=\left\{d_{\min }^{G_{0}} \geq n / 5\right\}$. We claim that it suffices to show

$$
\liminf _{n \rightarrow+\infty} \mathbb{E}_{G_{0} \sim \mathbb{P}_{1}}\left[\log \frac{\mathbb{P}_{2}\left[G=G_{0}\right]}{\mathbb{P}_{1}\left[G=G_{0}\right]} \mid \mathcal{E}\right] \geq 0
$$

Indeed since for any $G_{0}$ on $m$ edges (11.2) holds, $\log \frac{\mathbb{P}_{2}\left[G=G_{0}\right]}{\mathbb{P}_{1}\left[G=G_{0}\right]}$ is at most a quantity which is polynomial in $n$. Therefore

$$
\mathbb{E}_{G_{0} \sim \mathbb{P}_{1}}\left[\log \frac{\mathbb{P}_{2}\left[G=G_{0}\right]}{\mathbb{P}_{1}\left[G=G_{0}\right]}\right] \geq \mathbb{P}\left(\mathcal{E}^{c}\right) \operatorname{poly}(n)+\mathbb{P}(\mathcal{E}) \mathbb{E}_{G_{0} \sim \mathbb{P}_{1}}\left[\log \frac{\mathbb{P}_{2}\left[G=G_{0}\right]}{\mathbb{P}_{1}\left[G=G_{0}\right]} \mid \mathcal{E}\right] .
$$

By Lemma 11.2 we have $\mathbb{P}\left(\mathcal{E}^{c}\right)=2^{-\Omega(n)}$, and therefore indeed the condition (11.3) suffices for our result.

Now conditioning on $G_{0} \in \mathcal{E}$ we compute,

$$
\begin{aligned}
& \frac{\mathbb{P}_{2}\left[G=G_{0}\right]}{\mathbb{P}_{1}\left[G=G_{0}\right]}=\frac{1}{n} \sum_{v \in V\left(G_{0}\right)}\left(\frac{n-d^{G_{0}}(v)-1}{d^{G_{0}}(v)+k}\right)^{k}, \text { (by Lemma 11.1 ) } \\
& \geq \frac{1}{n} \sum_{v \in V\left(G_{0}\right)}\left(1+k \frac{n-2 d^{G_{0}}(v)-k-1}{d^{G_{0}}(v)+k}\right),\left(\text { using }(1+x)^{k} \geq 1+x k, x>-1\right)
\end{aligned}
$$

Therefore taking logarithms and conditional expectation it suffices to show

$$
\liminf _{n} \mathbb{E}_{G_{0} \sim \mathbb{P}_{1}}\left[\log \left(\frac{1}{n} \sum_{v \in V\left(G_{0}\right)}\left(1+k \frac{n-2 d^{G_{0}}(v)-k-1}{d^{G_{0}}(v)+k}\right)\right) \mid \mathcal{E}\right] \leq 0
$$


We use Jensen's inequality and that log is concave to conclude

$$
\mathbb{E}_{G_{0} \sim \mathbb{P}_{1}}\left[\log \left(\frac{1}{n} \sum_{v \in V\left(G_{0}\right)}\left(1+k \frac{n-2 d^{G_{0}}(v)-k-1}{d^{G_{0}}(v)+k}\right)\right) \mid \mathcal{E}\right]
$$

is at least

$$
\frac{1}{n} \sum_{v \in V\left(G_{0}\right)} \mathbb{E}_{G_{0} \sim \mathbb{P}_{1}}\left[\log \left(1+k \frac{n-2 d^{G_{0}}(v)-k-1}{d^{G_{0}}(v)+k}\right) \mid \mathcal{E}\right]
$$

Since the process is node-symmetric we conclude for an arbitrary fixed vertex $v$,

$\mathbb{E}_{G_{0} \sim \mathbb{P}_{1}}\left[\log \left(\frac{1}{n} \sum_{v \in V\left(G_{0}\right)}\left(1+k \frac{n-2 d^{G_{0}}(v)-k-1}{d^{G_{0}}(v)+k}\right)\right) \mid \mathcal{E}\right] \geq \mathbb{E}_{G_{0} \sim \mathbb{P}_{1}}\left[\log \left(1+k \frac{n-2 d^{G_{0}}(v)-k-1}{d^{G_{0}}(v)+k}\right) \mid \mathcal{E}\right]$.

Therefore it suffices to show

$$
\lim _{n \rightarrow+\infty} \mathbb{E}_{G_{0} \sim \mathbb{P}_{1}}\left[\left|\log \left(1+k \frac{n-2 d^{G_{0}}(v)-k-1}{d^{G_{0}}(v)+k}\right)\right| \mid \mathcal{E}\right]=0
$$

Using $\log (1+x) \leq x$ for $x>-1$, it suffices to show

$$
\lim _{n \rightarrow+\infty} \mathbb{E}_{G_{0} \sim \mathbb{P}_{1}}\left[\left|\left(k \frac{n-2 d^{G_{0}}(v)-k-1}{d^{G_{0}}(v)+k}\right)\right| \mid \mathcal{E}\right]=0 .
$$

Since we condition on the minimum degree being at least of order $n$, we have

$$
\begin{aligned}
\mathbb{E}_{G_{0} \sim \mathbb{P}_{1}}\left[\left|\left(k \frac{n-2 d^{G_{0}}(v)-k-1}{d^{G_{0}}(v)+k}\right)\right| \mid \mathcal{E}\right] & \leq O\left(\mathbb{E}_{G_{0} \sim \mathbb{P}_{1}}\left[\left|\left(k \frac{n-2 d^{G_{0}}(v)-k-1}{n}\right)\right| \mid \mathcal{E}\right]\right) \\
& =O\left(\frac{k}{n} \mathbb{E}_{G_{0} \sim \mathbb{P}_{1}}\left[\left|n-2 d^{G_{0}}(v)-k-1 \|\right| \mathcal{E}\right]\right) \\
& \leq O\left(\frac{k^{2}}{n}+\frac{k}{n} \mathbb{E}_{G_{0} \sim \mathbb{P}_{1}}\left[\left|n-2 d^{G_{0}}(v)\right| \mid \mathcal{E}\right]\right)
\end{aligned}
$$

Since $k=o(\sqrt{n})$ it suffices to prove

$$
\lim _{n} \frac{k}{n} \mathbb{E}_{G_{0} \sim \mathbb{P}_{1}}\left[\left|n-2 d^{G_{0}}(v)\right| \mid \mathcal{E}\right]=0 .
$$

Now

$$
\frac{k}{n} \mathbb{E}_{G_{0} \sim \mathbb{P}_{1}}\left[\left|n-2 d^{G_{0}}(v)\right|\right]=\mathbb{P}(\mathcal{E}) \frac{k}{n} \mathbb{E}_{G_{0} \sim \mathbb{P}_{1}}\left[\left|n-2 d^{G_{0}}(v)\right| \mid \mathcal{E}\right]+\mathbb{P}\left(\mathcal{E}^{c}\right) \frac{k}{n} \mathbb{E}_{G_{0} \sim \mathbb{P}_{1}}\left[\left|n-2 d^{G_{0}}(v)\right| \mid \mathcal{E}^{c}\right] .
$$

Since by Lemma 11.2, $\mathbb{P}\left(\mathcal{E}^{c}\right)=2^{-\Omega(n)}$ and $\left|n-2 d^{G_{0}}(v)\right|=O(n)$, almost surely, we conclude that to prove (11.5) it suffices to prove

$$
\lim _{n} \frac{k}{n} \mathbb{E}_{G_{0} \sim \mathbb{P}_{1}}\left[\mid n-2 d^{G_{0}}(v)\right]=0 .
$$


But the degree of a vertex of a uniform random graph with $m$ edges follows an hypergeometric distribution with population size $N, n-1$ success states and $m$ number of draws. In particular, it's mean is $\frac{m(n-1)}{N}=\frac{(n-1)(N / 2-k)}{N}=\frac{n-1}{2}\left(1+O\left(\frac{k}{N}\right)\right)$ and variance $O\left(\frac{m n N^{2}}{N^{3}}\right)=O\left(\frac{m n}{N}\right)=O(n)$. Therefore by triangle inequality,

$$
\begin{aligned}
\frac{k}{n} \mathbb{E}_{G_{0} \sim \mathbb{P}_{1}}\left[\left|n-2 d^{G_{0}}(v)\right|\right] & \leq \frac{k}{n} O\left(\left|n / 2-\mathbb{E}_{G_{0} \sim \mathbb{P}_{1}}\left(d^{G_{0}}(v)\right)\right|+\mathbb{E}_{G_{0} \sim \mathbb{P}_{1}}\left[\left|\mathbb{E}_{G_{0} \sim \mathbb{P}_{1}}\left(d^{G_{0}}(v)\right)-\left(d^{G_{0}}(v)\right)\right|\right]\right) \\
& \leq \frac{k}{n} O\left(\mid n / 2-\frac{n-1}{2}\left(1+O\left(\frac{k}{N}\right) \mid+\sqrt{\operatorname{Var}\left(d^{G_{0}}(v)\right)}\right), \quad\right. \text { (by Cauchy-Scharwz) } \\
& =\frac{k}{n} O\left(\frac{n k}{N}+\sqrt{n}\right) \\
& =O\left(\frac{k^{2}}{N}+\frac{k}{\sqrt{n}}\right) \\
& =o(1)\left(\text { since } N=\Theta\left(n^{2}\right), k^{2}=o(\sqrt{n})\right)
\end{aligned}
$$

The proof is complete.

Proof of Theorem 4.5. Set $\beta(\varepsilon)=\exp (\varepsilon)-1$.

Assume that there exists an $\varepsilon$-node-DP algorithm, $\mathcal{A}$, which can distinguish $P=G(n, m)$ and $Q=G(n, m+k)$ with probability bigger than $\exp (\varepsilon)-1>0$. Since $\mathcal{A}$ can distinguish between the models $P, Q$ there exists a query set $S$ such that for some $\delta>\exp (\varepsilon)-1$,

$$
\liminf _{n}\left|\mathbb{P}_{G \sim P}(\mathcal{A}(G) \in S)-\mathbb{P}_{H \sim Q}(\mathcal{A}(H) \in S)\right| \geq \delta>0
$$

Now in Proposition 4.4 we defined a disribution $R$ on unidrected graphs on $n$ vertices such that if $T$ is sampled from $R$ and $H$ from $Q, d_{v}(T, H)=1$, and furthermore

$$
\operatorname{TV}(R, P)=o(1)
$$

Since $\mathcal{A}$ is $\varepsilon$-node-DP, for any query $S_{0}$,

$$
\exp (-\varepsilon) \leq \frac{\mathbb{P}_{T \sim R}\left(\mathcal{A}(T) \in S_{0}\right)}{\mathbb{P}_{H \sim Q}\left(\mathcal{A}(H) \in S_{0}\right)} \leq \exp (\varepsilon)
$$

In particular, that implies

$$
\mathrm{TV}(R, Q) \leq \exp (\varepsilon)-1
$$

Using (11.8), (11.9) and triangle inequality we obtain

$$
\mathrm{TV}(Q, P) \leq o(1)+\exp (\varepsilon)-1 .
$$

Combined with (11.7) we conclude $\delta \leq \exp (\varepsilon)-1$, a contradiction.

\section{Acknowledgments}

A.S. was supported by NSF awards IIS-1447700 and AF-1763665, and a Sloan Foundation Research Award. I.Z. would like to thank Microsoft Research New England for providing exciting and hospitable enviroment during his summer internship in 2017 where part of this work was conducted. 


\section{References}

[1] E. Abbe and C. Sandon. Recovering communities in the general stochastic block model without knowing the parameters. arXiv:1503.00609, 2015.

[2] E. Abbe, A. S. Bandeira, and G. Hall. Exact recovery in the stochastic block model. arXiv:1405.3267, 2014.

[3] E. M. Airoldi, T. Costa, and S. Chan. A non-parametric perspective on network analysis: Theory and consistent estimation. In Advances in Neural Information Processing Systems (NIPS), volume 26, pages 692-700, 2013.

[4] D. Aldous. Representations for partially exchangeable arrays of random variables. J. Multivar. Anal., 11:581-598, 1981.

[5] P. J. Bickel and A. Chen. A nonparametric view of network models and newman-girvan and other modularities. Proceedings of the National Academy of Sciences, 106:21068-21073, 2009.

[6] P. J. Bickel, A. Chen, and E. Levina. The method of moments and degree distributions for network models. Annals of Statistics, 39(5):2280-2301, 2011.

[7] J. Blocki, A. Blum, A. Datta, and O. Sheffet. The Johnson-Lindenstrauss transform itself preserves differential privacy. In Symposium on Foundations of Computer Science (FOCS), pages 410-419, 2012. doi: 10.1109/FOCS.2012.67. URL http://dx.doi.org/10.1109/FOCS.2012.67.

[8] J. Blocki, A. Blum, A. Datta, and O. Sheffet. Differentially private data analysis of social networks via restricted sensitivity. In Innovations in Theoretical Computer Science (ITCS), pages $87-96,2013$.

[9] C. Borgs, J. T. Chayes, L. Lovász, V. Sós, and K. Vesztergombi. Counting graph homomorphisms. In Topics in Discrete Mathematics, pages 315-371. Springer, 2006.

[10] C. Borgs, J. T. Chayes, L. Lovász, V. Sós, and K. Vesztergombi. Convergent graph sequences I: Subgraph frequencies, metric properties, and testing. Advances in Math., 219:1801-1851, 2008.

[11] C. Borgs, J. T. Chayes, and L. Lovász. Moments of two-variable functions and the uniqueness of graph limits. Geometric And Functional Analysis, 19(6):1597-1619, 2010.

[12] C. Borgs, J. T. Chayes, L. Lovász, V. Sós, and K. Vesztergombi. Convergent graph sequences II: Multiway cuts and statistical physics. Ann. of Math., 176:151-219, 2012.

[13] C. Borgs, J. T. Chayes, H. Cohn, and Y. Zhao. An $L^{p}$ theory of sparse graph convergence I: limits, sparse random graph models, and power law distributions. arXiv:1401.2906, 2014.

[14] C. Borgs, J. T. Chayes, H. Cohn, and Y. Zhao. An $L^{p}$ theory of sparse graph convergence II: LD convergence, quotients, and right convergence. arXiv:1408.0744, 2014.

[15] C. Borgs, J. T. Chayes, and A. D. Smith. Private graphon estimation for sparse graphs. In Advances in Neural Information Processing Systems (NIPS), pages 1369-1377, 2015. 
[16] S. H. Chan and E. M. Airoldi. A consistent histogram estimator for exchangeable graph models. Journal of Machine Learning Research Workshop and Conference Proceedings, 32: 208-216, 2014.

[17] S. Chatterjee. Matrix estimation by universal singular value thresholding. Annals of Statistics, 43(1):177-214, 2015.

[18] S. Chen and S. Zhou. Recursive mechanism: towards node differential privacy and unrestricted joins. In ACM SIGMOD International Conference on Management of Data, pages 653-664, 2013.

[19] D. S. Choi, P. J. Wolfe, and E. M. Airoldi. Stochastic blockmodels with a growing number of classes. Biometrika, 99:273-284, 2012.

[20] J. Dall and M. Christensen. Random geometric graphs. Physical Review E, 2002.

[21] W. Day, N. Li, and M. Lyu. Publishing graph degree distribution with node differential privacy. In International Conference on Management of Data SIGMOD, pages 123-138, 2016. doi: 10.1145/2882903.2926745. URL http://doi.acm.org/10.1145/2882903.2926745.

[22] P. Diaconis and S. Janson. Graph limits and exchangeable random graphs. Rendiconti di Matematica, 28:33-61, 2008.

[23] C. Dwork, F. McSherry, K. Nissim, and A. Smith. Calibrating noise to sensitivity in private data analysis. In Theory of Cryptography Conference (TCC), pages 265-284, 2006.

[24] S. Galhotra, A. Mazumdar, S. Pal, and B. Saha. The geometric block model. In AAAI, 2018.

[25] C. Gao, Y. Lu, and H. H. Zhou. Rate-optimal graphon estimation. arXiv:1410.5837, 2014.

[26] E. N. Gilbert. Random plane networks. J. Soc. Indust. Appl. Math., (9):533-543, 1961.

[27] A. Gupta, A. Roth, and J. Ullman. Iterative constructions and private data release. In TCC, 2012.

[28] M. Hay, C. Li, G. Miklau, and D. Jensen. Accurate estimation of the degree distribution of private networks. In Int. Conf. Data Mining (ICDM), pages 169-178, 2009.

[29] M. Hay, V. Rastogi, G. Miklau, and D. Suciu. Boosting the Accuracy of Differentially Private Histograms Through Consistency. PVLDB, 3(1):1021-1032, 2010.

[30] P. Holland, K. Laskey, and S. Leinhardt. Stochastic blockmodels: First steps. Soc Netw, 5: 109-137, 1983.

[31] D. Hoover. Relations on probability spaces and arrays of random variables. Preprint, Institute for Advanced Study, Princeton, NJ, 1979.

[32] V. Karwa and A. Slavkovic. Inference using noisy degrees: Differentially private $\beta$-model and synthetic graphs. Ann. Statist., 44(1):87-112, 2016.

[33] V. Karwa and A. B. Slavkovic. Differentially private graphical degree sequences and synthetic graphs. In Privacy in Statistical Databases, pages 273-285, 2012. 
[34] V. Karwa, S. Raskhodnikova, A. D. Smith, and G. Yaroslavtsev. Private analysis of graph structure. ACM Trans. Database Syst., 39(3):22:1-22:33, 2014.

[35] V. Karwa, A. B. Slavkovic, and P. N. Krivitsky. Differentially private exponential random graphs. In Privacy in Statistical Databases (PSD), pages 143-155, 2014. doi: 10.1007/ 978-3-319-11257-2_12.

[36] S. P. Kasiviswanathan, K. Nissim, S. Raskhodnikova, and A. Smith. Analyzing graphs with node-differential privacy. In Theory of Cryptography Conference (TCC), pages 457-476, 2013.

[37] O. Klopp, A. B. Tsybakov, and N. Verzelen. Oracle inequalities for network models and sparse graphon estimation. Annals of Statistics Statistics, 45(1):316-354, 2017. doi: 10.1214/ 16-AOS1454.

[38] A. Korolova. Privacy violations using microtargeted ads: A case study. In IEEE International Conference on Data Mining Workshops, pages 474-482, 2010. doi: 10.1109/ICDMW.2010.137.

[39] P. Latouche and S. Robin. Bayesian model averaging of stochastic block models to estimate the graphon function and motif frequencies in a w-graph model. ArXiv:1310.6150, 2013.

[40] B.-R. Lin and D. Kifer. Information preservation in statistical privacy and Bayesian estimation of unattributed histograms. In ACM SIGMOD International Conference on Management of Data, pages 677-688, 2013.

[41] J. R. Lloyd, P. Orbanz, Z. Ghahramani, and D. M. Roy. Random function priors for exchangeable arrays with applications to graphs and relational data. In Advances in Neural Information Processing Systems (NIPS), volume 25, pages 1007-1015, 2012.

[42] L. Lovász and B. Szegedy. Limits of dense graph sequences. Journal of Combinatorial Theory, Series B, 96:933-957, 2006.

[43] W. Lu and G. Miklau. Exponential random graph estimation under differential privacy. In 20th ACM SIGKDD International Conference on Knowledge discovery and data mining, pages 921-930, 2014.

[44] A. McMillan and A. Smith. When is nontrivial estimation possible for graphons and stochastic block models? Information and Inference: A Journal of the IMA, 2017.

[45] D. J. Mir and R. N. Wright. A differentially private estimator for the stochastic kronecker graph model. In EDBT/ICDT Workshops, pages 167-176, 2012.

[46] A. Narayanan and V. Shmatikov. De-anonymizing social networks. In IEEE Symp. Security and Privacy, pages 173-187, 2009.

[47] K. Nissim, S. Raskhodnikova, and A. Smith. Smooth sensitivity and sampling in private data analysis. In Symp. Theory of Computing (STOC), pages 75-84, 2007.

[48] M. D. Penrose. Random geometric graphs. Oxford University Press, 2003.

[49] S. Raskhodnikova and A. D. Smith. Lipschitz extensions for node-private graph statistics and the generalized exponential mechanism. In Symposium on Foundations of Computer Science (FOCS), pages 495-504, 2016. doi: 10.1109/FOCS.2016.60. 
[50] V. Rastogi, M. Hay, G. Miklau, and D. Suciu. Relationship privacy: output perturbation for queries with joins. In Symp. Principles of Database Systems (PODS), pages 107-116, 2009.

[51] K. Rohe, S. Chatterjee, and B. Yu. Spectral clustering and the high-dimensional stochastic blockmodel. Ann. Statist., 39(4):1878-1915, 082011.

[52] A. R. S. Janson, T. Luczak. Random Graphs. Wiley-Interscience, 2011.

[53] M. Tang, D. L. Sussman, and C. E. Priebe. Universally consistent vertex classification for latent positions graphs. Annals of Statistics, 41(3):1406-1430, 06 2013. doi: 10.1214/13-AOS1112.

[54] P. Wolfe and S. C. Olhede. Nonparametric graphon estimation. arXiv:1309.5936, 2013.

[55] Q. Xiao, R. Chen, and K. Tan. Differentially private network data release via structural inference. In The 20th ACM SIGKDD International Conference on Knowledge Discovery and Data Mining (KDD), New York, NY, USA, pages 911-920, 2014.

[56] J. J. Yang, Q. Han, and E. M. Airoldi. Nonparametric estimation and testing of exchangeable graph models. In Proceedings of 17th AISTATS (JMLR: WECP volume 33), 2014. 